ALEA, Lat. Am. J. Probab. Math. Stat. 16, 605-632 (2019)

\title{
Quadratic fluctuations of the symmetric simple exclusion
}

\section{Patrícia Gonçalves and Milton Jara}

Center for Mathematical Analysis, Geometry and Dynamical Systems

Instituto Superior Técnico, Universidade de Lisboa,

Av. Rovisco Pais, 1 ,

1049-001 Lisboa, Portugal.

E-mail address: pgoncalves@tecnico.ulisboa.pt

$U R L:$ http://patriciamath.wix.com/patricia

IMPA

Estrada Dona Castorina 110,

CEP 22460,

Rio de Janeiro, Brasil.

E-mail address: mjara@impa.br

URL: https://impa.br/page-pessoas/milton-jara/

\begin{abstract}
We introduce a two-dimensional, distribution-valued field, which we call the quadratic field, associated with the one-dimensional Ornstein-Uhlenbeck process and we prove that the stationary quadratic fluctuations of the simple exclusion process, in the diffusive scaling, converge to this quadratic field. Moreover, we prove that this quadratic field evaluated at the diagonal corresponds to the Wick-renormalized square of the Ornstein-Uhlenbeck process, and we use this new representation in order to prove some small and large-time properties of it.
\end{abstract}

\section{Introduction}

In recent years, scaling limits of nonlinear and/or singular functionals of stochastic lattice models have attracted a lot of attention. Just to give a couple of examples, we mention the extensive studies of the KPZ universality class (see Corwin, 2012 for a review) and Gaussian multiplicative chaos associated to Liouiville quantum gravity (see Rhodes and Vargas, 2014 for a review). In Hairer (2014) the author has proposed a general framework (the so-called theory of regularity structures) in order to deal with ill-posed stochastic PDE's on which the trouble comes from a nonlinear term (like in the KPZ or the stochastic Allen-Cahn equations)

Received by the editors May 17th, 2018; accepted March 28, 2019.

2010 Mathematics Subject Classification. 60K35, 35K55.

Key words and phrases. Quadratic fluctuations, simple exclusion.

M.J. would like to thank the warm hospitality at the Institut de Mathématiques de Marseille and to Etienne Pardoux for valuable comments and for helping with the proof of Theorem 3.9. 
or from a singular linear term (like in the parabolic Anderson model). The theory of regularity structures allows to make sense of troublesome equations in a meaningful way. Moreover, various scaling limits of stochastic lattice models on which these singular and/or nonlinear observables play an important role should be given in terms of solutions to these equations. However, aside from models on which a great deal of integrability is present (the term stochastic integrability was coined in Spohn (2012)) or models starting from an explicitly known stationary measure, the question of convergence of nonlinear fluctuations of stochastic lattice models is basically open; see however Gonçalves and Jara (2014); Gonçalves et al. (2015, 2017); Franco et al. (2016); Blondel et al. (2016); Gonçalves et al. (2019+); Diehl et al. (2017) and references therein.

One of the main ingredients of the theory of regularity structures consists in making sense a priori of enough nonlinear and/or singular observables of solutions of linear stochastic PDE's. In the case of the KPZ equation Hairer (2013), one starts with the solution of the Ornstein-Uhlenbeck equation

$$
\partial_{t} \mathcal{Y}_{t}=\Delta \mathcal{Y}_{t}+\frac{1}{\sqrt{2}} \nabla \omega_{t}
$$

where $\omega_{t}$ is a space-time white noise and one tries to make sense of various nonlinear functionals of it (the twelve tree-labeled processes in Hairer, 2013). To avoid uncomfortable issues arising from the lack of compactness, the author of Hairer (2013) restricts to the circle $\mathbb{T}$.

The simplest of these tree-labeled processes corresponds to $\mathcal{Y}_{t}(x)^{2}$. Since $\mathcal{Y}_{t}$ turns out to be a distribution, it is far from clear how to define $\mathcal{Y}_{t}(x)^{2}$. Let us consider the stationary situation, in which for any fixed time $t, \mathcal{Y}_{t}$ is a spatial white noise of variance $\chi=\frac{1}{4}$. The simplest choice should be to take an approximation of the identity $\iota_{\varepsilon}(x)$ centered at $x \in \mathbb{T}$, and to consider $\mathcal{Y}_{t}(x)^{2}$ as the limit of $\mathcal{Y}_{t}\left(\iota_{\varepsilon}(x)\right)^{2}$ as $\varepsilon \rightarrow 0$ in some sense. It turns out that this plan can be formalized after a Wick renormalization: when integrated in time and space against a smooth test function, $\mathcal{Y}_{t}\left(\iota_{\varepsilon}(x)\right)^{2}-\frac{\chi}{\varepsilon}$ has a meaningful limit as $\varepsilon \rightarrow 0$.

Of course, if one wants to use Hairer (2013) in order to obtain a unique solution of the KPZ equation, there are still 11 processes to go, but in this paper we just focus on $\mathcal{Y}_{t}(x)^{2}$ (we do not claim we can treat the other 11 processes!). Up to our knowledge, this squared field was first considered in Assing (2007), where the convergence of space-time fluctuations of two-point functions to the squared field of the Ornstein-Uhlenbeck process was obtained. A more general, different proof was implicitly obtained in Gonçalves and Jara (2014) and explicitly stated in Gonçalves and Jara (2013), as a part of a program towards the derivation of the KPZ equation from general stochastic lattice models.

In this paper we propose a new, different approach in order to define the field $\mathcal{Y}_{t}(x)^{2}$. The quadratic field associated with $\mathcal{Y}_{t}$ is the two-dimensional process formally defined by

$$
Q_{t}(x, y)=\mathcal{Y}_{t}(x) \mathcal{Y}_{t}(y) .
$$

In order to define this object in a weak sense, some care needs to be taken at the diagonal; the simplest choice is to take $Q_{t}(x, x)=0$, (anyway $Q_{t}$ is just a formal 
object). Blindly applying Itô's formula we see that $Q_{t}$ satisfies an equation of the form

$$
d Q_{t}=\Delta Q_{t}+d \mathcal{W}_{t}
$$

where $\mathcal{W}_{t}$ is a distribution-valued martingale that can be computed in terms of the Ornstein-Uhlenbeck process $\mathcal{Y}_{t}$ and the noise $d \omega_{t}$. It turns out that this equation is well-posed and solutions of it can be constructed straightforwardly. Let $\iota_{\varepsilon}(x, y)$ be a two-dimensional approximation of the identity. Let $f: \mathbb{T} \rightarrow \mathbb{R}$ be a mean-zero regular function and consider the process

$$
\mathcal{A}_{t}^{\varepsilon}(f)=\int_{0}^{t} Q_{s}\left(\left(f^{\prime} \otimes \delta\right) * \iota_{\varepsilon}\right) d s,
$$

where $\left(f^{\prime} \otimes \delta\right)$, and $\iota_{\varepsilon}$ are defined in (2.9) and (2.11), respectively.

It turns out that the process $\mathcal{A}_{t}^{\varepsilon}$ has a non-trivial limit $\mathcal{A}_{t}$, as $\varepsilon \rightarrow 0$ and this limit coincides with the process constructed in Definition 1 of Assing (2007). The main difference is that now we obtain the squared process as a singular linear observable of the solution of a reasonably well-behaved stochastic PDE. The main advantage of this representation is that by solving a simple Laplace problem, we can obtain various properties of the squared process in a more or less straightforward way.

In order to keep the paper at a reasonable length, we focus on two issues related to the quadratic field $Q_{t}$, and we keep the model as simple as possible. First we show that the quadratic fluctuations of the simple exclusion process converge to the quadratic field $Q_{t}$. It is well known that the simple exclusion process is integrable in the sense that various quantities of interest, among them $n$-point correlation functions, can be computed almost explicitly. We do not take full advantage of this feature. Given the technical Boltzmann-Gibbs principle for granted, a careful reading of our proof shows that this convergence result can be extended for the speed-change exclusion processes considered in Gonçalves and Jara (2014). This technical principle has been proved in De Masi et al. (1984).

Then we obtain short-time and long-time properties of the process $\mathcal{A}_{t}$, using the construction outlined above. These properties have not been obtained before, and they are good examples of the advantages of our construction when compared with the previous ones.

Outline: This paper is organized as follows. In Section 2 we introduce the notations and we state our main results. More precisely, we introduce the exclusion process and the related fluctuation fields. On the way, we introduce various topological notations which are needed to handle distribution-valued processes and also state the main results of the article, namely Theorems 2.4, 2.5, 2.7 and 2.8.

In Section 3 we prove Theorem 2.4. In Section 3.1 we introduce some exponential martingales associated with the process. In Section 3.2, we show that the discrete quadratic fields form a relatively compact sequence of distributions with respect to the $J_{1}$-Skorohod topology on the space of distribution-valued càdlàg paths.

In the rest of Section 3 we show that the discrete quadratic fields converge to the unique stationary solution of equation (1.2). The proof is divided in three parts. 
First limit points exist due to tightness. Then, in Section 3.3, we show that any limit point is continuous; this will be important later when characterizing some martingales in terms of quadratic variations. In Section 3.4 we show that various martingales associated with the discrete quadratic fields have limit points which are martingales. This shows that any limit point of the discrete quadratic fields satisfies a martingale formulation of (1.2).

In Section 3.5 we show that this martingale problem has a unique solution, which closes the proof of the convergence result. In Section 4 we obtain information about the singular field $\mathcal{A}_{t}(f)$ for small and large times $t$. For small times $t$ we show that $\mathcal{A}_{t}(f)$ looks like a fractional Brownian motion of Hurst exponent 3/4. This result, combined with the results of Gonçalves and Jara (2013), proves the following claim: fluctuations of local linear observables of conservative systems (like occupation times) have the same qualitative behavior as fluctuations of global quadratic observables (like the field $\mathcal{A}_{t}(f)$ ).

For large times $t$, since we are working on a compact setting, any quantity is diffusive. However, the value of the corresponding diffusion coefficient is not obvious. We show that this diffusion coefficient is given by the energy associated with the fractional Laplacian of order $1 / 2$, plus a finite-size effect.

\section{The model and statement of results}

2.1. The symmetric simple exclusion process. Let $\Omega_{n}=\{0,1\}^{\mathbb{T}_{n}}$ be the state space of a continuous-time Markov chain where $\mathbb{T}_{n}=\frac{1}{n} \mathbb{Z} / \mathbb{Z}$ is the discrete circle with $n$ points. The elements of $\Omega_{n}$ are denoted by $\eta=\left\{\eta(x) ; x \in \mathbb{T}_{n}\right\}$ and we call them configurations while the elements of $\mathbb{T}_{n}$ are called sites. We consider the set $\mathbb{T}_{n}$ embedded in the continuous circle $\mathbb{T}=\mathbb{R} / \mathbb{Z}$. We say that two sites $x, y \in \mathbb{T}_{n}$ are neighbors if $|y-x|=\frac{1}{n}$.

To each pair $\{x, y\}$ of neighbors we attach a Poisson clock of rate $n^{2}$. Each Poisson clock is independent of the others. Each time a Poisson clock rings, we exchange the occupation numbers of the corresponding pair of neighbors. For each $\eta \in \Omega_{n}$ and each $x, y \in \mathbb{T}_{n}$, let $\eta^{x, y} \in \Omega_{n}$ denote the configuration obtained from $\eta$ by exchanging the occupation numbers at $x$ and $y$, that is,

$$
\eta^{x, y}(z)= \begin{cases}\eta(y), & z=x \\ \eta(x), & z=y \\ \eta(z), & z \neq x, y\end{cases}
$$

The Markov chain $\left\{\eta_{t}^{n} ; t \geq 0\right\}$ has infinitesimal generator denoted by $L_{n}$ which is given on $f: \Omega_{n} \rightarrow \mathbb{R}$ by

$$
L_{n} f(\eta)=n^{2} \sum_{x \in \mathbb{T}_{n}} \nabla_{x, x+1 / n} f(\eta)
$$

where $\nabla_{x, x+1 / n} f(\eta)=f\left(\eta^{x, x+1 / n}\right)-f(\eta)$ for any $x \in \mathbb{T}_{n}$ and any $\eta \in \Omega_{n}$.

We say that a site $x$ is occupied by a particle at time $t \geq 0$ if $\eta_{t}^{n}(x)=1$. If $\eta_{t}^{n}(x)=0$, we say that the site $x$ is empty at time $t \geq 0$. With this convention about particles and empty sites (or holes), the dynamics of $\left\{\eta_{t}^{n} ; t \geq 0\right\}$ has the following interpretation. Each particle tries to jump to each of its two neighbours with exponential rate $n^{2}$. At each attempt, it verifies whether the destination site is empty, on which case it jumps to it. Otherwise the particle stays where it is. This particle interpretation gives the name simple exclusion process to the family 
of processes $\left\{\eta_{t}^{n} ; t \geq 0\right\}$. Note that particles are neither created nor annihilated by this dynamics. By reversibility, it is easy to check that, for any $\ell \in\{0,1, \ldots, n\}$, the uniform measures $\nu_{n, \ell}$ on the spaces

$$
\Omega_{n, \ell}=\left\{\eta \in \Omega_{n} ; \sum_{x \in \mathbb{T}_{n}} \eta(x)=\ell\right\}
$$

are invariant with respect to the dynamics of $\left\{\eta_{t}^{n} ; t \geq 0\right\}$. Checking the irreducibility of the sets $\Omega_{n, \ell}$ with respect to the dynamics, it can be concluded that $\nu_{n, \ell}$ is actually ergodic under the evolution of $\left\{\eta_{t}^{n} ; t \geq 0\right\}$ for any $\ell$. The product measures $\nu_{\rho}$ given by

$$
\nu_{\rho}(\eta)=\prod_{x \in \mathbb{T}_{n}}\{\rho \eta(x)+(1-\rho)(1-\eta(x))\}
$$

are invariant and reversible under $\left\{\eta_{t}^{n} ; t \geq 0\right\}$ for any $\rho \in[0,1]$.

From now on we start the process $\left\{\eta_{t}^{n} ; t \geq 0\right\}$ from the invariant measure $\nu_{\rho}$ for $\rho=\frac{1}{2}$. In order to simplify the notation from now on we use the notation $\bar{\eta}$ to denote the centered r.v. $\eta$, that is $\bar{\eta}=\eta-\frac{1}{2}$.

To avoid uninteresting topological issues, we fix $T>0$ and we restrict the process $\left\{\eta_{t}^{n} ; t \geq 0\right\}$ to the interval $[0, T]$. We denote by $\mathbb{P}_{n}$ the distribution of $\left\{\eta_{t}^{n} ; t \in[0, T]\right\}$ on the space $\mathcal{D}\left([0, T] ; \Omega_{n}\right)$ of càdlàg trajectories from $[0, T]$ to $\Omega_{n}$, and we denote by $\mathbb{E}_{n}$ the expectation with respect to $\mathbb{P}_{n}$. The expectation with respect to $\nu_{n, \ell}$ will be denoted by $E_{n, \ell}$, and the expectation with respect to $\nu_{\rho}$ will be denoted by $E_{\rho}$.

2.2. The fluctuation fields. In order to define the fluctuation fields associated with the process $\left\{\eta_{t}^{n} ; t \in[0, T]\right\}$ in a proper way, we need to introduce further notations and some topologies.

For $\ell=1,2$, let $\mathbb{T}^{\ell}$ be the $\ell$-dimensional torus. Let $C^{\infty}\left(\mathbb{T}^{\ell}\right)$ denote the set of infinitely often differentiable functions $f: \mathbb{T}^{\ell} \rightarrow \mathbb{R}$. The space $C^{\infty}\left(\mathbb{T}^{\ell}\right)$ is a Polish space with respect to the topology generated by the metric $d: C^{\infty}\left(\mathbb{T}^{\ell}\right) \times C^{\infty}\left(\mathbb{T}^{\ell}\right) \rightarrow$ $[0, \infty)$ given by

$$
d(f, g)=\sum_{n \in \mathbb{N}_{0}} \frac{1}{2^{n}} \min \left\{1,\left\|f^{(n)}-g^{(n)}\right\|_{\infty}\right\} .
$$

Here $f^{(n)}$ denotes the $n$-th derivative of $f$, which is a function in the case $\ell=1$ and an $n$-dimensional, symmetric tensor in the case $\ell=2$.

The space $D^{\prime}\left(\mathbb{T}^{\ell}\right)$ is the set of linear, continuous functions $\varphi: C^{\infty}\left(\mathbb{T}^{\ell}\right) \rightarrow \mathbb{R}$. In other words, $D^{\prime}\left(\mathbb{T}^{\ell}\right)$ is the topological dual of $C^{\infty}\left(\mathbb{T}^{\ell}\right)$, which is known in the literature as the space of distributions in $\mathbb{T}^{\ell}$ and functions $f \in C^{\infty}\left(\mathbb{T}^{\ell}\right)$ are called test functions.

We will define two distribution-valued processes associated with $\left\{\eta_{t}^{n} ; t \in[0, T]\right\}$; one with values in $D^{\prime}(\mathbb{T})$ and another one with values in $D^{\prime}\left(\mathbb{T}^{2}\right)$. It turns out that the spaces $D^{\prime}(\mathbb{T}), D^{\prime}\left(\mathbb{T}^{2}\right)$ equipped with the weak topology are Polish spaces. For $\ell=1,2$, we denote the space of càdlàg trajectories equipped with the $J_{1}$-Skorohod topology by $\mathcal{D}\left([0, T] ; D^{\prime}\left(\mathbb{T}^{\ell}\right)\right)$.

Definition 2.1 (Density fluctuation field).

Let $\left\{\mathcal{Y}_{t}^{n} ; t \in[0, T]\right\}$ denote the $D^{\prime}(\mathbb{T})$-valued process given on $f \in C^{\infty}(\mathbb{T})$ and $t \in[0, T]$ by

$$
\mathcal{Y}_{t}^{n}(f)=\frac{1}{\sqrt{n}} \sum_{x \in \mathbb{T}_{n}} f(x) \bar{\eta}_{t}^{n}(x)
$$


This process has been extensively studied, and in particular a scaling limit for it is available. Let $\omega$ be a standard space-time white noise in $\mathbb{T} \times[0, T]$, that is, a Gaussian distribution such that

$$
\int_{0}^{T} \int_{\mathbb{T}} f(x, t) \omega(d x d t)
$$

has a Gaussian law of mean 0 and variance $\int_{0}^{T} \int_{\mathbb{T}} f^{2} d x d t$ for any $f \in C^{\infty}(\mathbb{T} \times[0, T])$.

Proposition 2.2 (De Masi et al., 1984). The sequence $\left\{\mathcal{Y}_{t}^{n} ; t \in[0, T]\right\}_{n \geq 1}$ converges in distribution, with respect to the $J_{1}$-topology of $\mathcal{D}\left([0, T] ; D^{\prime}(\mathbb{T})\right)$, as $n \rightarrow$ $+\infty$, to the stationary solution of the infinite-dimensional Ornstein-Uhlenbeck equation

$$
\partial_{t} \mathcal{Y}_{t}=\Delta \mathcal{Y}_{t}+\frac{1}{\sqrt{2}} \nabla \omega
$$

where $\omega$ is a space-time white noise.

Definition 2.3 (Quadratic fluctuation field).

Let $\left\{Q_{t}^{n} ; t \in[0, T]\right\}$ denote the $D^{\prime}\left(\mathbb{T}^{2}\right)$-valued process given on $f \in C^{\infty}\left(\mathbb{T}^{2}\right)$ by

$$
Q_{t}^{n}(f)=\frac{1}{n} \sum_{\substack{x, y \in \mathbb{T}_{n} \\ x \neq y}} f(x, y) \bar{\eta}_{t}^{n}(x) \bar{\eta}_{t}^{n}(y)
$$

for any $t \in[0, T]$.

Without loss of generality, from now on and up to the end of the article we will assume that any test function $f \in C^{\infty}\left(\mathbb{T}^{2}\right)$ is symmetric, and note that for any antisymmetric function $f \in C^{\infty}\left(\mathbb{T}^{2}\right), Q_{t}^{n}(f)=0$.

The process $\left\{Q_{t}^{n} ; t \in[0, T]\right\}$ is called the quadratic fluctuation field associated with the process $\left\{\eta_{t}^{n} ; t \in[0, T]\right\}$. Our aim will be to obtain the scaling limit of the process $\left\{Q_{t}^{n} ; t \in[0, T]\right\}$, as $n \rightarrow \infty$.

Theorem 2.4. Let $\left\{\mathcal{W}_{t} ; t \in[0, T]\right\}$ be the martingale process defined as

$$
\mathcal{W}_{t}(f)=\frac{1}{\sqrt{2}} \int_{0}^{t} \int_{\mathbb{T}}\left\{\mathcal{Y}_{s}\left(\partial_{2} f(\cdot, x)\right)+\mathcal{Y}_{s}\left(\partial_{1} f(x, \cdot)\right)\right\} \omega(d x d s)
$$

for any test function $f \in C^{\infty}\left(\mathbb{T}^{2}\right)$. Above $\mathcal{Y}_{s}$ is the stationary solution of the infinite-dimensional Ornstein-Uhlenbeck equation given in (2.7). The process $\left\{Q_{t}^{n} ; t \in[0, T]\right\}$ converges in distribution, with respect to the $J_{1}$-topology of $\mathcal{D}\left([0, T] ; D^{\prime}\left(\mathbb{T}^{2}\right)\right)$, as $n \rightarrow+\infty$, to the stationary solution of the equation

$$
d Q_{t}=\Delta Q_{t} d t+d \mathcal{W}_{t}
$$

In Section 3, we will prove the previous theorem using the standard three-steps method to get convergence in distribution of stochastic processes, namely, we first prove tightness of the sequence of processes in a suitable topology, then we deduce some properties of the possible limit points using the approximating processes, and then we show that these aforementioned properties characterize the limit point in a unique way. 
2.3. A singular fluctuation field. The main purpose of the introduction of the fluctuation field $\left\{Q_{t} ; t \in[0, T]\right\}$ given in Theorem 2.4, is the study of the quadratic field $\left\{\mathcal{A}_{t} ; t \in[0, T]\right\}$ formally defined as follows. For a function $f \in C^{\infty}(\mathbb{T})$, we denote by $f \otimes \delta$ the distribution in $\mathbb{T}^{2}$ given by

$$
\langle f \otimes \delta, g\rangle=\int_{\mathbb{T}} f(x) g(x, x) d x,
$$

where $g: \mathbb{T}^{2} \rightarrow \mathbb{R}$. In other words, $f \otimes \delta(x, y)=f(x) \delta(x, y)$, where $\delta(x, y)$ represents the uniform measure on the diagonal $\left\{(x, y) \in \mathbb{T}^{2} ; x=y\right\}$. Then, we formally define $\mathcal{A}_{t}(f)$ as

$$
\mathcal{A}_{t}(f)=\int_{0}^{t} Q_{s}\left(f^{\prime} \otimes \delta\right) d s,
$$

where $Q_{s}$ is the process given in Theorem 2.4. Of course, it is not clear at all whether this definition makes any sense. The simplest idea one can use is to approximate the singular object $f^{\prime} \otimes \delta$ by a sequence of more regular functions. Let us consider the approximation of the identity $\left\{\iota_{\varepsilon} ; 0<\varepsilon<1\right\}$ given by

$$
\iota_{\varepsilon}(x, y)=\frac{1}{4 \varepsilon^{2}} \mathbb{1}_{|x| \leq \varepsilon} \mathbb{1}_{|y| \leq \varepsilon} .
$$

This approximation of the identity is not the smoothest one we can use, but it is very convenient and we will see later on why. The following theorem explains how to define the singular process $\left\{\mathcal{A}_{t} ; t \in[0, T]\right\}$.

Theorem 2.5. Let $\left\{\iota_{\varepsilon} ; \varepsilon \in(0,1)\right\}$ be the approximation of the identity in $\mathbb{T}^{2}$ defined in (2.11). Let $\left\{\mathcal{A}_{t}^{\varepsilon} ; t \in[0, T]\right\}$ be the $D^{\prime}(\mathbb{T})$-valued process defined as

$$
\mathcal{A}_{t}^{\varepsilon}(f)=\int_{0}^{t} Q_{s}\left(\left(f^{\prime} \otimes \delta\right) * \iota_{\varepsilon}\right) d s
$$

for any $f \in C^{\infty}(\mathbb{T})$ and any $t \in[0, T]$. Then, $\left\{\mathcal{A}_{t}^{\varepsilon} ; t \in[0, T]\right\}$ converges in distribution with respect to the uniform topology, as $\varepsilon \rightarrow 0$, to a well-defined, $D^{\prime}(\mathbb{T})$-valued process $\left\{\mathcal{A}_{t} ; t \in[0, T]\right\}$.

We observe that above we used the test function $\left(f^{\prime} \otimes \delta\right) * \iota_{\varepsilon}$ which is not in $C^{\infty}\left(\mathbb{T}^{2}\right)$. Nevertheless, by an approximation procedure similar to the one used for $\mathcal{Y}_{s}\left(\iota_{\varepsilon}\right)$ in Gonçalves and Jara (2014), we can still make sense of $Q_{s}\left(\left(f^{\prime} \otimes \delta\right) * \iota_{\varepsilon}\right)$. Last theorem was proved in Gonçalves and Jara (2013), although the proof there is very different from the proof we will present here. Moreover, the proof that we present here has one important advantage: as we will see, it gives a more explicit construction of the process $\left\{\mathcal{A}_{t} ; t \in[0, T]\right\}$, which allows to obtain various properties of it. For completeness, we present the following convergence result, obtained in Gonçalves and Jara (2013):

Proposition 2.6. Let $\left\{\mathcal{A}_{t}^{n} ; t \in[0, T]\right\}$ be the $D^{\prime}(\mathbb{T})$-valued process defined by

$$
\mathcal{A}_{t}^{n}(f)=\int_{0}^{t} \sum_{x \in \mathbb{T}_{n}} f^{\prime}(x) \bar{\eta}_{s}^{n}(x) \bar{\eta}_{s}^{n}\left(x+\frac{1}{n}\right) d s
$$

for any $f \in C^{\infty}(\mathbb{T})$. The process $\left\{\mathcal{A}_{t}^{n} ; t \in[0, T]\right\}$ converges in distribution, as $n \rightarrow \infty$, to the process $\left\{\mathcal{A}_{t} ; t \in[0, T]\right\}$ defined in $(2.10)$.

Note that considering intervals of arbitrary length $T$, we can assume that $\left\{\mathcal{A}_{t} ; t \geq\right.$ $0\}$ is a well-defined process. As we mentioned in the introduction, our construction 
allows to obtain some properties of the field $\left\{\mathcal{A}_{t} ; t \geq 0\right\}$. The short-time properties of $\left\{\mathcal{A}_{t} ; t \geq 0\right\}$ are given by the following theorem:

Theorem 2.7. As $\varepsilon \rightarrow 0$, for each $f \in C^{\infty}(\mathbb{T})$, the field

$$
\left\{\varepsilon^{-3 / 4} \mathcal{A}_{\varepsilon t}(f) ; t \geq 0\right\}
$$

converges in distribution, with respect to the uniform topology, to a stationary Gaussian process $\left\{\mathcal{B}_{t}(f) ; t \geq 0\right\}$ with covariance given by

$E\left[\mathcal{B}_{t}(f) \mathcal{B}_{s}(f)\right]=\frac{4}{3 \sqrt{\pi}}(-1+\sqrt{2})\left\{t^{3 / 2}+s^{3 / 2}-|t-s|^{3 / 2}\right\} \int_{\mathbb{T}} f(x)(-\Delta f(x)) d x$.

Note the similarity of the covariance formula of (2.14) with the covariance of the process obtained in Theorem 2.5 of Gonçalves and Jara (2013). As far as we understand, this result has not been predicted in the literature.

For large times $t$, we only know the limiting variance of $\mathcal{A}_{t}(f)$ :

Theorem 2.8. There exists a convolution operator $K: L^{2}(\mathbb{T}) \rightarrow L^{2}(\mathbb{T})$ such that for any $f \in C^{\infty}(\mathbb{T})$ it holds that

$$
\lim _{t \rightarrow \infty} \frac{1}{t} \mathbb{E}\left[\mathcal{A}_{t}(f)^{2}\right]=\int_{\mathbb{T}} f(x)\left(-\frac{1}{2}(-\Delta)^{\frac{1}{2}} f(x)+K f(x)\right) d x .
$$

Above the operator $(-\Delta)^{\frac{1}{2}}$ is the fractional Laplacian of exponent $\frac{1}{2}$ defined, by means of its Fourier representation as

$$
-\widehat{(-\Delta)^{1} / 2} f(k)=-2 \pi|k| \widehat{f}(k)
$$

\section{Proof of Theorem 2.4}

3.1. Auxiliary martingales. Let $F: \Omega_{n} \times[0, T] \rightarrow \mathbb{R}$ be a smooth function on the time variable. Then, the process

$$
\exp \left\{F_{t}\left(\eta_{t}^{n}\right)-F_{0}\left(\eta_{0}^{n}\right)-\int_{0}^{t} \exp \left\{-F_{s}\left(\eta_{s}^{n}\right)\right\}\left(\partial_{s}+L_{n}\right) \exp \left\{F_{s}\right\}\left(\eta_{s}^{n}\right) d s\right\}
$$

is a positive martingale with unit expectation. We will apply this formula for the function $F=\theta Q^{n}(f)$, where $\theta \in \mathbb{R}$ and for a symmetric function $f: \mathbb{T}_{n}^{2}=$ $\mathbb{T}_{n} \times \mathbb{T}_{n} \rightarrow \mathbb{R}$ and for $\eta \in \Omega_{n}$,

$$
Q^{n}(f)(\eta)=\frac{1}{n} \sum_{\substack{x, y \in \mathbb{T}_{n} \\ x \neq y}} f(x, y) \bar{\eta}(x) \bar{\eta}(y) .
$$

For $n \in \mathbb{N}$ and $f \in C^{\infty}\left(\mathbb{T}^{2}\right)$, define $Q_{t}^{n}(f)=Q^{n}(f)\left(\eta_{t}^{n}\right)$. For each $\theta \in \mathbb{R}$ and each $f \in C^{\infty}\left(\mathbb{T}^{2}\right)$, let $\left\{\mathcal{M}_{t}^{\theta, n}(f) ; t \in[0, T]\right\}$ denote the martingale given by

$$
\mathcal{M}_{t}^{\theta, n}(f)=\exp \left\{\theta\left(Q_{t}^{n}(f)-Q_{0}^{n}(f)\right)-\int_{0}^{t} \exp \left\{-\theta Q_{s}^{n}(f)\right\} L_{n} \exp \left\{\theta Q_{s}^{n}(f)\right\} d s\right\}
$$

for any $t \in[0, T]$. Let us compute the integral term above. Note that for any function $f: \Omega_{n} \rightarrow \mathbb{R}$

$$
\exp \{-f\} L_{n} \exp \{f\}=\sum_{x \in \mathbb{T}_{n}} n^{2}\left(\exp \left\{\nabla_{x, x+1 / n} f\right\}-1\right)
$$


and that

$$
\nabla_{x, x+1 / n} Q^{n}(f)(\eta)=\frac{1}{n} \xi^{n}(f ; x)(\eta)
$$

where

$$
\xi^{n}(f ; x)(\eta)=\frac{2}{n} \sum_{z \neq x, x+1 / n} \bar{\eta}(z) f_{x}^{n}(z)\left(\eta(x)-\eta\left(x+\frac{1}{n}\right)\right)
$$

where above, for $x \in \mathbb{T}, n \in \mathbb{N}$ and $f \in C^{\infty}\left(\mathbb{T}^{2}\right), f_{x}^{n}: \mathbb{T} \rightarrow \mathbb{R}$ is defined as

$$
f_{x}^{n}(z)=n\left(f\left(z, x+\frac{1}{n}\right)-f(z, x)\right) .
$$

Note that the difference $f\left(z, x+\frac{1}{n}\right)-f(z, x)$ is of order $\frac{1}{n}$. In fact, since $f \in C^{\infty}\left(\mathbb{T}^{2}\right)$, this difference is approximated by $\frac{1}{n} \partial_{2} f(z, x)$, with an error term of order $\frac{1}{n^{2}}$. In particular, we have the a priori bound

$$
\left|\xi_{t}^{n}(f ; x)(\eta)\right| \leq c_{1}(f)
$$

for any $n \in \mathbb{N}$, any $x \in \mathbb{T}_{n}$ and any $t \in[0, T]$, where $\xi_{t}^{n}(f ; x)=\xi^{n}(f ; x)\left(\eta_{t}^{n}\right)$. Since the measure $\nu_{\frac{1}{2}}$ is of product form, we have a better bound for the second moment of $\xi_{t}^{n}(f ; x)$ :

$$
\mathbb{E}_{n}\left[\xi_{t}^{n}(f ; x)^{2}\right] \leq \frac{c_{2}(f)}{n}
$$

for any $n \in \mathbb{N}$, any $x \in \mathbb{T}_{n}$ and any $t \in[0, T]$. We point out here that the a priori bound (3.6) will be very useful, since the term $\xi_{t}^{n}(f ; x)$ will actually appear in a double exponential, and therefore moment bounds will not be as useful. Going back to the computation of the martingale, we see that

$$
\mathcal{M}_{t}^{\theta, n}(f)=\exp \left\{\theta\left(Q_{t}^{n}(f)-Q_{0}^{n}(f)\right)-\int_{0}^{t} \sum_{x \in \mathbb{T}_{n}} n^{2}\left(\exp \left\{\frac{\theta}{n} \xi_{s}^{n}(f ; x)\right\}-1\right) d s\right\} .
$$

Now we note that for any $n \in \mathbb{N}$, the process

$$
\left.\frac{\partial^{\ell}}{\partial \theta^{\ell}} \mathcal{M}_{t}^{\theta, n}(f)\right|_{\theta=0}
$$

is also a martingale. Then, for $\ell=1$, the process $\left\{\mathcal{W}_{t}^{n}(f) ; t \in[0, T]\right\}$ given by

$$
\mathcal{W}_{t}^{n}(f)=Q_{t}^{n}(f)-Q_{0}^{n}(f)-\int_{0}^{t} \sum_{x \in \mathbb{T}_{n}} n \xi_{s}^{n}(f ; x) d s
$$

is a martingale. The previous equality is a consequence of a change of variables. Now we compute the term on the right hand side of last expression. Note that

$$
\begin{aligned}
& \sum_{x \in \mathbb{T}_{n}} n \xi^{n}(f ; x)(\eta) \\
& =2 \sum_{x, z \neq x, x+\frac{1}{n}} \bar{\eta}(z) f_{x}^{n}(z)\left(\eta(x)-\eta\left(x+\frac{1}{n}\right)\right) \\
& =2 \sum_{x, z} \bar{\eta}(z) f_{x}^{n}(z)\left(\eta(x)-\eta\left(x+\frac{1}{n}\right)\right) \\
& -2 \sum_{x} \bar{\eta}(x) f_{x}^{n}(x)\left(\eta(x)-\eta\left(x+\frac{1}{n}\right)\right) \\
& -2 \sum_{x} \bar{\eta}\left(x+\frac{1}{n}\right) f_{x}^{n}(x+1)\left(\eta(x)-\eta\left(x+\frac{1}{n}\right)\right)
\end{aligned}
$$




$$
\begin{aligned}
& =\frac{1}{n} \sum_{x \neq z \in \mathbb{T}_{n}} \bar{\eta}(x) \bar{\eta}(z) \Delta_{n} f(x, z) \\
& -2 n \sum_{x \in \mathbb{T}_{n}} \bar{\eta}(x) \bar{\eta}\left(x+\frac{1}{n}\right)\left(f\left(x+\frac{1}{n}, x+\frac{1}{n}\right)+f(x, x)-2 f\left(x, x+\frac{1}{n}\right)\right),
\end{aligned}
$$

where for $f \in C^{\infty}\left(\mathbb{T}^{2}\right)$, the operator $\Delta_{n} f: \mathbb{T}_{n} \times \mathbb{T}_{n} \rightarrow \mathbb{R}$ is defined as

$$
\begin{aligned}
\Delta_{n} f(x, z) & =n^{2}\left(f\left(x+\frac{1}{n}, z\right)+f\left(x-\frac{1}{n}, z\right)-2 f(x, z)\right) \\
& +n^{2}\left(f\left(x, z+\frac{1}{n}\right)+f\left(x, z-\frac{1}{n}\right)-2 f(x, z)\right) .
\end{aligned}
$$

In other words, $\Delta_{n} f$ is a discrete version of the Laplacian $\Delta f=\partial_{11} f+\partial_{22} f$. Note that the first term on the right-hand side of (3.10) can be written as $Q^{n}\left(\Delta_{n} f\right)$. With respect to the measure $\nu_{\frac{1}{2}}$, the expectation of the second term on the righthand side of (3.10) is equal to 0. Due to the smoothness and the symmetry of $f$ it is also simple to check that its variance with respect to $\nu_{\frac{1}{2}}$ vanishes as $n \rightarrow \infty$. In other words, only the first term on the right-hand side of (3.10) will be relevant one when considering scaling limits.

Taking $\ell=2$ in (3.8), we see that the process

$$
\mathcal{W}_{t}^{n}(f)^{2}-\int_{0}^{t} \sum_{x \in \mathbb{T}_{n}} \xi_{s}^{n}(f ; x)^{2} d s
$$

is also a martingale. In other words, the predictable quadratic variation of the martingale process $\left\{\mathcal{W}_{t}^{n}(f) ; t \in[0, T]\right\}$ is given by

$$
\left\langle\mathcal{W}^{n}(f)\right\rangle_{t}=\int_{0}^{t} \sum_{x \in \mathbb{T}_{n}} \xi_{s}^{n}(f ; x)^{2} d s .
$$

Note that (3.7) implies a bound of the form $\mathbb{E}_{n}\left[\left\langle\mathcal{W}^{n}(f)\right\rangle_{t}\right] \leq c(f) t$ for the quadratic variation process, and therefore a moment bound of the form $\mathbb{E}_{n}\left[\mathcal{W}_{t}^{n}(f)^{2}\right] \leq c(f) t$ for the martingale process $\left\{\mathcal{W}_{t}^{n}(f) ; t \in[0, T]\right\}$.

3.2. Tightness. In this section we prove tightness of the sequence of processes $\left\{Q_{t}^{n} ; t \in[0, T]\right\}_{n \in \mathbb{N}}$. The first step is to reduce the problem from distribution-valued processes to real-valued processes. This is done throught the so-called Mitoma's criterion.

Proposition 3.1 (Mitoma's criterion, see Mitoma, 1983).

The sequence of $D^{\prime}\left(\mathbb{T}^{2}\right)$-valued processes $\left\{Q_{t}^{n} ; t \in[0, T]\right\}_{n \in \mathbb{N}}$ is tight in $\mathcal{D}\left([0, T] ; D^{\prime}\left(\mathbb{T}^{2}\right)\right)$ if and only if the sequence $\left\{Q_{t}^{n}(f) ; t \in[0, T]\right\}_{n \in \mathbb{N}}$ is tight in $\mathcal{D}([0, T] ; \mathbb{R})$ for any $f \in C^{\infty}\left(\mathbb{T}^{2}\right)$. Moreover, if any limit point of $\left\{Q_{t}^{n}(f) ; t \in\right.$ $[0, T]\}_{n \in \mathbb{N}}$ is supported on continuous, real-valued trajectories for any $f \in C^{\infty}\left(\mathbb{T}^{2}\right)$, then any limit point of $\left\{Q_{t}^{n} ; t \in[0, T]\right\}_{n \in \mathbb{N}}$ is supported on continuous $D^{\prime}\left(\mathbb{T}^{2}\right)$-valued trajectories.

Applying this criterion, we see that it is enough to prove tightness for the process $Q_{t}^{n}(f)$ given in (3.9). We note that we will not prove tightness directly for the process $\left\{Q_{t}^{n}(f) ; t \in[0, T]\right\}$, but for each one of the process appearing in the decomposition given in (3.9). 
3.2.1. Tightness of $\left\{Q_{0}^{n}(f) ; n \in \mathbb{N}\right\}$. Taking characteristic functions, it is easy to see that $Q_{0}^{n}(f)$ converges to a Gaussian random variable of mean 0 and variance

$$
\frac{1}{16} \iint_{\mathbb{T}^{2}} f(x, y)^{2} d x d y
$$

Note that this is also true for the sequence $\left\{Q_{t}^{n}(f) ; n \in \mathbb{N}\right\}$ for any time $t \in[0, T]$. Since any convergent sequence is tight, we are done with this term.

\subsubsection{Tightness of the integral term. Let}

$$
\mathcal{I}_{t}^{n}(f)=\int_{0}^{t} \sum_{x \in \mathbb{T}_{n}} n \xi_{s}^{n}(f ; x) d s
$$

To prove tightness of $\left\{\mathcal{I}_{t}^{n}(f) ; t \in[0, T]\right\}_{n \in \mathbb{N}}$, we use the following criterion, see, for example, Theorem 2.3 of Ferrari et al. (1988).

Proposition 3.2. The sequence of processes $\left\{\mathcal{I}_{t}^{n}(f) ; t \in[0, T]\right\}_{n \in \mathbb{N}}$ is tight with respect to the uniform topology of $\mathcal{C}([0, T] ; \mathbb{R})$ if

$$
\sup _{n \in \mathbb{N}} \sup _{0 \leq t \leq T} \mathbb{E}_{n}\left[\left(\sum_{x \in \mathbb{T}_{n}} n \xi_{t}^{n}(f ; x)\right)^{2}\right]<\infty
$$
for

By the stationarity of the process $\left\{\eta_{t}^{n} ; t \in[0, T]\right\}$, it is enough to get a bound

$$
E_{\frac{1}{2}}\left[\left(\sum_{x \in \mathbb{T}_{n}} n \xi^{n}(f ; x)(\eta)\right)^{2}\right]
$$

Recall (3.10) and the comments thereafter. Since $f$ is smooth and symmetric , there exists a constant $C(f)$ which does not depend on $n$ such that

$$
E_{\frac{1}{2}}\left[\left(n \sum_{x \in \mathbb{T}_{n}} \bar{\eta}(x) \bar{\eta}\left(x+\frac{1}{n}\right)\left(f\left(x+\frac{1}{n}, x+\frac{1}{n}\right)+f(x, x)-2 f\left(x, x+\frac{1}{n}\right)\right)\right)^{2}\right] \leq C(f) .
$$

On the other hand,

$$
E_{\frac{1}{2}}\left[\left(\frac{1}{n} \sum_{x \neq z \in \mathbb{T}_{n}} \bar{\eta}(x) \bar{\eta}(z) \Delta_{n} f(x, z)\right)^{2}\right] \leq \frac{1}{n^{2}} \sum_{x, z \in \mathbb{T}_{n}}\left(\Delta_{n} f(x, z)\right)^{2},
$$

and due to the smoothness of $f$, the right-hand side of this inequality can also be bounded by a (maybe different) constant $C(f)$ which does not depend on $n$. We conclude that the sequence of processes $\left\{\mathcal{I}_{t}^{n}(f) ; t \in[0, T]\right\}_{n \in \mathbb{N}}$ is tight with respect to the uniform topology of $\mathcal{C}([0, T] ; \mathbb{R})$.

3.2.3. Tightness of the martingale term. To prove tightness of the sequence $\left\{\mathcal{W}_{t}^{n}(f) ; t \in[0, T]\right\}_{n \in \mathbb{N}}$, we use the following well-known criterion, see, for example, Theorem 2.3 in Ferrari et al. (1988).

Proposition 3.3. The sequence of real-valued processes $\left\{\mathcal{W}_{t}^{n}(f) ; t \in[0, T]\right\}_{n \in \mathbb{N}}$ is tight in $\mathcal{D}([0, T] ; \mathbb{R})$ if

$$
\sup _{n \in \mathbb{N}} \int_{0}^{T} \mathbb{E}_{n}\left[\left(\sum_{x \in \mathbb{T}_{n}} \xi_{t}^{n}(f ; x)^{2}\right)^{2}\right] d t<\infty
$$


Let us show that this supremum is finite. By stationarity, we just need to show that

$$
\left.\sup _{n \in \mathbb{N}} E_{\frac{1}{2}}\left[\left(\sum_{x \in \mathbb{T}_{n}} \xi^{n}(f ; x)(\eta)\right)^{2}\right)^{2}\right]<\infty .
$$

Now, using the Cauchy-Schwarz inequality, we need to show that

$$
\sup _{n \in \mathbb{N}} n \sum_{x \in \mathbb{T}_{n}} E_{\frac{1}{2}}\left[\xi^{n}(f ; x)(\eta)^{4}\right]<\infty
$$

A simple computation shows that

$$
n \sum_{x \in \mathbb{T}_{n}} E_{\frac{1}{2}}\left[\xi^{n}(f ; x)(\eta)^{4}\right] \leq \frac{C}{n^{2}} \sum_{x, z \in \mathbb{T}_{n}}\left(f_{x}^{n}(z)\right)^{4}
$$

and, due to the smoothness of $f$, last expression converges, as $n \rightarrow+\infty$, to

$$
\iint\left(\partial_{2} f(z, x)\right)^{4} d z d x
$$

which shows that the supremum in Proposition 3.3 is finite. We conclude that the martingale sequence $\left\{\mathcal{W}_{t}^{n}(f) ; t \in[0, T]\right\}_{n \in \mathbb{N}}$ is tight in $\mathcal{D}([0, T] ; \mathbb{R})$.

Up to here, we have proved tightness of $\left\{Q_{t}^{n} ; t \in[0, T]\right\}_{n \in \mathbb{N}}$ by combining Mitoma's criterion with the tightness of the processes appearing in the decomposition (3.9). We remark that we do not know whether the limit points of $\left\{\mathcal{W}_{t}^{n}(f) ; t \in[0, T]\right\}_{n \in \mathbb{N}}$ are continuous, and in particular, we do not know whether the limit points of $\left\{Q_{t}^{n} ; t \in[0, T]\right\}$ are concentrated on continuous, $D^{\prime}\left(\mathbb{T}^{2}\right)$-valued trajectories. We will address this question below, when discussing the characterization of such limit points.

3.3. Characterization of limit points: the process $\mathcal{W}_{t}$. In the previous section, we showed tightness of the sequence $\left\{Q_{t}^{n} ; t \in[0, T]\right\}_{n \in \mathbb{N}}$. Let $\left\{Q_{t} ; t \in[0, T]\right\}$ be one of such limit points. For ease of notation, the superscript $n$ will denote along this section, the subsequence for which the process $\left\{Q_{t}^{n} ; t \in[0, T]\right\}$ converges to $\left\{Q_{t} ; t \in\right.$ $[0, T]\}$. Without loss of generality, we can assume that the process $\left\{\mathcal{W}_{t}^{n}(f) ; t \in\right.$ $[0, T]\}$ converges to a process $\left\{\mathcal{W}_{t}(f) ; t \in[0, T]\right\}$, along the subsequence $n$, for any $f \in C^{\infty}\left(\mathbb{T}^{2}\right)$. Interpreting $\left\{\left\langle\mathcal{W}^{n}(f)\right\rangle_{t} ; t \in[0, T]\right\}$ as an integral process, we see that the estimates used to prove tightness of $\left\{\mathcal{W}_{t}^{n}(f) ; t \in[0, T]\right\}$ are exactly the ones needed to apply the tightness criterion given in Proposition 3.2 to $\left\{\left\langle\mathcal{W}^{n}(f)\right\rangle_{t} ; t \in\right.$ $[0, T]\}$. We leave the details to the reader. Taking a further sub-subsequence if necessary, we can assume that along $n$, the process $\left\{\left\langle\mathcal{W}^{n}(f)\right\rangle_{t} ; t \in[0, T]\right\}$ converges in distribution to some process $\left\{\langle\mathcal{W}(f)\rangle_{t} ; t \in[0, T]\right\}$ Without further comments, taking sub-subsequences if necessary, we will assume that any process we need to define is convergent along the subsequence $n$. Note that based solely on convergence in distribution, we can not argue that $\left\{\langle\mathcal{W}(f)\rangle_{t} ; t \in[0, T]\right\}$ is the quadratic variation of $\left\{\mathcal{W}_{t}(f) ; t \in[0, T]\right\}$. In fact, we do not even know whether $\left\{\mathcal{W}_{t}(f) ; t \in[0, T]\right\}$ is a martingale.

In this section we prove the following result, which aims at answering the questions raised in the previous paragraph.

Theorem 3.4. For any function $f \in C^{\infty}\left(\mathbb{T}^{2}\right)$, there exists a (strictly positive) constant $\theta(f)$ such that the process $\left\{\mathcal{M}_{t}^{\theta}(f) ; t \in[0, T]\right\}$ given by

$$
\mathcal{M}_{t}^{\theta}(f)=\exp \left\{\theta \mathcal{W}_{t}(f)-\frac{1}{2} \theta^{2}\langle\mathcal{W}(f)\rangle_{t}\right\}
$$


is a martingale for any $\theta \in \mathbb{R}$ such that $|\theta| \leq \theta(f)$.

The proof of last result is done in two steps. First we prove that the exponential martingales given in (3.2) converge in distribution along the subsequence $n$ to $\mathcal{M}_{t}^{\theta}(f)$ and then we prove that they are uniformly integrable. The two results combined give that the limit $\mathcal{M}_{t}^{\theta}(f)$ is a martingale and we are done.

To prove the convergence, first, note that we do not know whether the martingales $\mathcal{M}_{t}^{\theta, n}(f)$ are tight, and therefore we cannot say anything about convergence. The following simple Taylor estimate will be very useful.

For any $u \in \mathbb{R}$ and any $\ell \in \mathbb{N}$,

$$
\left|\exp \{u\}-\sum_{i=0}^{\ell-1} \frac{u^{i}}{i !}\right| \leq \frac{1}{\ell !}|u|^{\ell} \exp \{|u|\} .
$$

Fix $f \in C^{\infty}\left(\mathbb{T}^{2}\right)$ and $\theta \in \mathbb{R}$. We will use this estimate for $u=\frac{\theta}{n} \xi_{s}^{n}(f ; x)$ and $\ell=4$. Recall (3.6). We have that

$$
\begin{aligned}
& \left|\exp \left\{\frac{\theta}{n} \xi_{s}^{n}(f ; x)\right\}-1-\frac{\theta}{n} \xi_{s}^{n}(f ; x)-\frac{\theta^{2}}{2 n^{2}} \xi_{s}^{n}(f ; x)^{2}-\frac{\theta^{3}}{6 n^{3}} \xi_{s}^{n}(f ; x)^{3}\right| \\
& \leq \frac{\theta^{4}}{24 n^{4}} c_{1}(f)^{4} \exp \left\{\theta c_{1}(f)\right\} .
\end{aligned}
$$

Therefore,

$$
\mathcal{M}_{t}^{\theta, n}(f)=\exp \left\{\theta \mathcal{W}_{t}^{n}(f)-\frac{\theta^{2}}{2}\left\langle\mathcal{W}^{n}(f)\right\rangle_{t}-\int_{0}^{t} \frac{\theta^{3}}{6 n} \sum_{x \in \mathbb{T}_{n}} \xi_{s}^{n}(f ; x)^{3} d s+\mathcal{R}_{t}^{\theta, n}(f)\right\},
$$

where the error term $\mathcal{R}_{t}^{\theta, n}(f)$ satisfies

$$
\left|\mathcal{R}_{t}^{\theta, n}\right| \leq \frac{\theta^{4} c_{1}(f)^{4} t}{24 n^{2}} \exp \left\{\theta c_{1}(f)\right\}
$$

and, in particular, it goes to 0 , uniformly in $n$. Note that

$$
\left|\frac{1}{n} \sum_{x \in \mathbb{T}_{n}} \xi_{s}^{n}(f ; x)^{3}\right| \leq c_{1}(f)^{3}, \quad \mathbb{E}_{n}\left[\left|\frac{1}{n} \sum_{x \in \mathbb{T}_{n}} \xi_{s}^{n}(f ; x)^{3}\right|\right] \leq \frac{c_{1}(f) c_{2}(f)}{n}
$$

and we see that the cubic term converges to 0 in probability as $n \rightarrow \infty$. Looking into (3.14) and using the remark before Corollary 3.7, we have just showed that along the subsequence $n$, for any fixed $t \in[0, T]$ and any $f \in C^{\infty}\left(\mathbb{T}^{2}\right)$, the random variable $\mathcal{M}_{t}^{\theta, n}(f)$ converges in distribution to

$$
\mathcal{M}_{t}^{\theta}(f)=\exp \left\{\theta \mathcal{W}_{t}(f)-\frac{1}{2} \theta^{2}\langle\mathcal{W}(f)\rangle_{t}\right\}
$$

Note that although we have not proved that the convergence holds at the level of processes, we do know that $\left\{\mathcal{M}_{t}^{\theta}(f) ; t \in[0, T]\right\}$ is a well-defined process with trajectories in $\mathcal{D}([0, T] ; \mathbb{R})$. The argument above can also be used to obtain convergence of any finite-dimensional distributions.

In order to prove that $\left\{\mathcal{M}_{t}^{\theta}(f) ; t \in[0, T]\right\}$ is a martingale, it is enough to show that the sequence $\left\{\mathcal{M}_{T}^{\theta, n}(f) ; n \in \mathbb{N}\right\}$ is uniformly integrable. It is here where we will take full advantage of the decomposition (3.14). In fact, the error term and the cubic term in (3.14) are uniformly bounded in $n$ by a deterministic constant. Therefore, we can neglect them. Moreover, the term $\left\langle\mathcal{W}^{n}(f)\right\rangle_{t}$ is non-negative and it appears with a minus sign. Therefore, we can also neglect it. We are left to prove the uniform integrability of the sequence $\left\{\exp \left\{\theta \mathcal{W} \mathcal{W}_{T}^{n}(f)\right\} ; n \in \mathbb{N}\right\}$. The simplest criterion for uniform integrability is a uniform $L^{p}\left(\mathbb{P}_{n}\right)$-bound for some 
$p>1$. In other words, we want to estimate $\mathbb{E}_{n}\left[\exp \left\{p \theta \mathcal{W}_{T}^{n}(f)\right\}\right]$ for some $p>1$. Recall that $\mathbb{E}_{n}\left[\mathcal{M}_{T}^{\theta, n}(f)\right]=1$. We will use the elementary estimate, which is a trivial consequence of the Cauchy-Schwarz inequality,

$$
E[\exp \{X\}] \leq E[\exp \{2(X-Y)\}]^{1 / 2} E[\exp \{2 Y\}]^{1 / 2}
$$

for $X=p \theta \mathcal{W}_{T}^{n}(f)$ and

$$
Y=p^{2} \theta^{2}\left\langle\mathcal{W}^{n}(f)\right\rangle_{T}+\int_{0}^{T} \frac{2 p^{3} \theta^{3}}{3 n} \sum_{x \in \mathbb{T}_{n}} \xi_{t}^{n}(f ; x)^{3} d t-\frac{1}{2} \mathcal{R}_{T}^{2 p \theta, n}(f) .
$$

With these choices, we get the bound

$$
\mathbb{E}_{n}\left[\exp \left\{p \theta \mathcal{W}_{T}^{n}(f)\right\}\right] \leq \mathbb{E}_{n}[\exp \{2 Y\}]^{\frac{1}{2}} .
$$

We can again neglect the cubic term and the error term in (3.16), since they are uniformly bounded in $n$ by a deterministic constant. Therefore, uniform integrability of $\left\{\mathcal{M}_{T}^{\theta, n}(f) ; n \in \mathbb{N}\right\}$ will be proved if we can show that

$$
\sup _{n \in \mathbb{N}} \mathbb{E}_{n}\left[\exp \left\{2 p^{2} \theta^{2}\left\langle\mathcal{W}^{n}(f)\right\rangle_{T}\right\}\right]<\infty .
$$

Recall the definition of $\left\langle\mathcal{W}^{n}(f)\right\rangle_{T}$ from (3.12) and rewrite it as

$$
\left\langle\mathcal{W}^{n}(f)\right\rangle_{T}=\frac{1}{T} \int_{0}^{T} \sum_{x \in \mathbb{T}_{n}} T \xi_{t}^{n}(f ; x)^{2} d t .
$$

By the convexity of the exponential function,

$$
\mathbb{E}_{n}\left[\exp \left\{2 p^{2} \theta^{2}\left\langle\mathcal{W}^{n}(f)\right\rangle_{T}\right\}\right] \leq \frac{1}{T} \int_{0}^{T} \mathbb{E}_{n}\left[\exp \left\{\beta \sum_{x \in \mathbb{T}_{n}} \xi_{t}^{n}(f ; x)^{2}\right\}\right] d t,
$$

where $\beta=2 p^{2} \theta^{2} T$. Therefore, we just need to show that

$$
\sup _{n \in \mathbb{N}} E_{\frac{1}{2}}\left[\exp \left\{\beta \sum_{x \in \mathbb{T}_{n}} \xi^{n}(f ; x)^{2}\right\}\right]<\infty .
$$

Now, using the crude exponential Hölder estimate

$$
E\left[\exp \left\{\sum_{i} X_{i}\right\}\right] \leq \prod_{i} E\left[\exp \left\{n X_{i}\right\}\right]^{1 / n}
$$

and we bound the expectation in (3.17) from above by

$$
\prod_{x \in \mathbb{T}_{n}} E_{\frac{1}{2}}\left[\exp \left\{\beta n \xi^{n}(f ; x)^{2}\right\}\right]^{\frac{1}{n}}
$$

From now on we fix $x \in \mathbb{T}_{n}$ and our next goal is to bound from above the previous expectation. Since for any $x \in \mathbb{T}_{n},|\eta(x)| \leq 1$, we have that

$$
E_{\frac{1}{2}}\left[\exp \left\{\beta n \xi^{n}(f ; x)^{2}\right\}\right] \leq E_{\frac{1}{2}}\left[\exp \left\{\beta n\left(\frac{2}{n} \sum_{z \neq x, x+1 / n} \bar{\eta}(z) f_{x}^{n}(z)\right)^{2}\right\}\right] .
$$

Note that $f_{x}^{n}(z)$ is a discrete approximation of the partial derivative $\partial_{2} f(z, x)$. Now we use the following simple observation. For any non-negative random variable $X$ and any regular function $f$,

$$
E[f(X)]=f(0)+\int_{0}^{\infty} f^{\prime}(t) P(X \geq t) d t .
$$


Therefore, for $f(t)=\exp \{t\}$, the right hand-side of (3.18) is bounded from above by

$$
1+\int_{0}^{\infty} \exp \{t\} P_{\frac{1}{2}}\left(\frac{2}{n}\left|\sum_{z \neq x, x+1 / n} \bar{\eta}(z) f_{x}^{n}(z)\right| \geq \sqrt{\frac{t}{\beta n}}\right) d t .
$$

In order to bound last probability we use Hoeffding's inequality.

Proposition 3.5 (Hoeffding's inequality).

There exist constants $C_{H}, c_{H}$ such that for any $n \in \mathbb{N}$, for any $A \subseteq \mathbb{T}_{n}$ and for any $g: A \rightarrow \mathbb{R}$,

$$
P_{\frac{1}{2}}\left(\left|\sum_{z \in A} \bar{\eta}(z) g(z)\right| \geq \lambda\right) \leq C_{H} \exp \left\{-\frac{c_{H} \lambda^{2}}{\sum_{z \in A} g(z)^{2}}\right\} .
$$

Taking $\lambda=\sqrt{\frac{t}{\beta n}}$ in (3.19), the integral above is bounded by

$$
\int_{0}^{\infty} C_{H} \exp \left\{-t\left(\frac{c_{H}}{\beta n \sum_{z \neq x, x+1 / n}\left(f_{x}^{n}(z)\right)^{2}}-1\right)\right\} d t .
$$

By the smoothness of $f$, there exists a constant $c_{2}(f)$ such that

$$
\frac{4}{n} \sum_{z \neq x, x+1 / n}\left(f_{x}^{n}(z)\right)^{2} \leq c_{2}(f)
$$

for any $n \in \mathbb{N}$ and any $x \in \mathbb{T}_{n}$. Therefore, for any $\beta$ such that $\beta<\frac{c_{H}}{c_{2}(f)}$,

$$
\begin{aligned}
& \int_{0}^{\infty} C_{H} \exp \left\{-t\left(\frac{c_{H}}{\frac{4 \beta}{n} \sum_{z \neq x, x+1 / n}\left(f_{x}^{n}(z)\right)^{2}}-1\right)\right\} d t \\
& \leq \int_{0}^{\infty} C_{H} \exp \left\{-t\left(\frac{c_{H}}{\beta c_{2}(f)}-1\right)\right\} d t=C_{H}\left\{\frac{c_{H}}{c_{H}-\beta c_{2}(f)}-1\right\}
\end{aligned}
$$

and, since $\beta=2 p^{2} \theta^{2} T$, we conclude that

$$
E_{\frac{1}{2}}\left[\exp \left\{2 p^{2} \theta^{2} T \sum_{x \in \mathbb{T}_{n}} \xi^{n}(f ; x)^{2}\right\}\right] \leq 1+C_{H}\left\{\frac{c_{H}}{c_{H}-2 p^{2} \theta^{2} T c_{2}(f)}-1\right\}
$$

for any $n \in \mathbb{N}$ and $\theta \in \mathbb{R}$ small enough; and this proves (3.17) as desired. We conclude that the sequence $\left\{\mathcal{M}_{T}^{\theta, n}(f) ; n \in \mathbb{N}\right\}$ is uniformly integrable for any $\theta \in \mathbb{R}$ satisfying

$$
|\theta| \leq \sqrt{\frac{c_{H}}{2 T c_{2}(f)}}
$$

and this ends the proof.

Note that this theorem implies that the processes

$$
\left\{\left.\frac{\partial^{\ell}}{\partial \theta^{\ell}} \mathcal{M}_{t}^{\theta}(f)\right|_{\theta=0} ; t \in[0, T]\right\}
$$

are martingales for any $\ell \in \mathbb{N}_{0}$. In particular, considering the cases $\ell=1,2$ we get the following result.

Corollary 3.6. For any function $f \in C^{\infty}\left(\mathbb{T}^{2}\right)$, the process $\left\{\mathcal{W}_{t}(f) ; t \in[0, T]\right\}$ is a martingale of quadratic variation $\left\{\langle\mathcal{W}(f)\rangle_{t} ; t \in[0, T]\right\}$. 
Finally, by noting that,

$$
\sup _{0 \leq t \leq T}\left|\mathcal{W}_{t}^{n}(f)-\mathcal{W}_{t^{-}}^{n}(f)\right|=\sup _{0 \leq t \leq T}\left|Q_{t}^{n}(f)-Q_{t^{-}}^{n}(f)\right|
$$

and since at each time there is at most one jump, the previous display is of order $O\left(\frac{1}{n}\right)$. Moreover, from (3.9) and taking the limit in $n \rightarrow+\infty$, we can conclude that the process $\left\{\mathcal{W}_{t}(f) ; t \in[0, T]\right\}$ has continuous trajectories. This answers a question raised in the previous section about the continuity of $\left\{Q_{t} ; t \in[0, T]\right\}$ and we state it as a corollary.

Corollary 3.7. Any limit point of the sequence of $D^{\prime}\left(\mathbb{T}^{2}\right)$-valued processes $\left\{Q_{t}^{n} ; t \in\right.$ $[0, T]\}_{n \in \mathbb{N}}$ is concentrated on continuous, $D^{\prime}\left(\mathbb{T}^{2}\right)$-valued tractories.

3.4. Characterization of the martingale processes. Our next goal will be to characterize the quadratic variation of the martingale $\left\{\mathcal{W}_{t}(f) ; t \in[0, T]\right\}$ in terms of the process $\left\{\mathcal{Y}_{t} ; t \in[0, T]\right\}$. More precisely, in this section we prove the next result.

Theorem 3.8. For any $f \in C^{\infty}\left(\mathbb{T}^{2}\right)$ and any $t \in[0, T]$,

$$
\langle\mathcal{W}(f)\rangle_{t}=2 \int_{0}^{t} \int_{\mathbb{T}} \mathcal{Y}_{s}\left(g_{x}\right)^{2} d x d s
$$

where $g_{x}: \mathbb{T} \rightarrow \mathbb{R}$ is defined as $g_{x}(z)=\partial_{2} f(z, x)$ and $\mathcal{Y}_{s}$ is the solution of $(2.7)$.

Before proving the theorem we recall that by definition $Q_{t}(f)=Q_{t}\left(f_{s}\right)$, where

$$
f_{s}(x, y)=\frac{1}{2}(f(x, y)+f(y, x))
$$

is the symmetric part of $f$. The expression for $\langle\mathcal{W}(f)\rangle_{t}$ does not look very symmetric in the coordinates $(x, y)$. Note that for any smooth, symmetric function $f: \mathbb{T}^{2} \rightarrow$ $\mathbb{R}, \partial_{2} f(x, y)=\partial_{1} f(y, x)$. This relation allows us to write $\langle\mathcal{W}(f)\rangle_{t}$ in the more symmetric way

$$
\langle\mathcal{W}(f)\rangle_{t}=\int_{0}^{t} \int_{\mathbb{T}}\left(\mathcal{Y}_{s}\left(\partial_{1} f(x, \cdot)\right)^{2}+\mathcal{Y}_{s}\left(\partial_{2} f(\cdot, x)\right)^{2}\right) d x d s
$$

Proof: Recall (3.10) and note that the second sum on the right-hand side of (3.10) converges to 0 in $L^{2}$, while the first sum is equal to $Q_{s}^{n}(\Delta f)$ plus an error term which also goes to 0 in $L^{2}$. Taking the limit in (3.9) through the subsequence $n$, we get the identity

$$
\mathcal{W}_{t}(f)=Q_{t}(f)-Q_{0}(f)-\int_{0}^{t} Q_{s}(\Delta f) d t
$$

valid for any $f \in C^{\infty}\left(\mathbb{T}^{2}\right)$ and any $t \in[0, T]$. From (3.4), we see that

$$
\begin{aligned}
\xi_{t}^{n}(f ; x) & -\frac{2}{\sqrt{n}}\left(\eta_{t}^{n}(x)-\eta_{t}^{n}\left(x+\frac{1}{n}\right)\right) \mathcal{Y}_{t}^{n}\left(f_{x}^{n}\right) \\
= & -2\left\{\bar{\eta}_{t}^{n}(x) \bar{\eta}_{t}^{n}\left(x+\frac{1}{n}\right)-\frac{1}{4}\right\}\left(f\left(x+\frac{1}{n}, x+\frac{1}{n}\right)+f(x, x)-2 f\left(x, x+\frac{1}{n}\right)\right) .
\end{aligned}
$$

In particular, there exists a constant $c_{3}(f)$ such that

$$
\left|\xi_{t}^{n}(f ; x)-\frac{2}{\sqrt{n}}\left(\eta_{t}^{n}(x)-\eta_{t}^{n}\left(x+\frac{1}{n}\right)\right) \mathcal{Y}_{t}^{n}\left(f_{x}^{n}\right)\right| \leq \frac{c_{3}(f)}{n^{2}},
$$


for any $n \in \mathbb{N}$, any $x \in \mathbb{T}_{n}$ and any $t \in[0, T]$. Using the simple identity $a^{2}-b^{2}=$ $2 a(a-b)-(a-b)^{2}$, we see that

$$
\left|\sum_{x \in \mathbb{T}_{n}}\left\{\xi_{t}^{n}(f ; x)^{2}-\frac{4}{n} c_{x}\left(\eta_{t}^{n}\right) \mathcal{Y}_{t}^{n}\left(f_{x}^{n}\right)^{2}\right\}\right| \leq \frac{c_{3}(f)^{2}}{n^{3}}+\frac{c_{1}(f) c_{3}(f)}{n},
$$

where

$$
c_{x}\left(\eta_{t}^{n}\right):=\left(\eta_{t}^{n}(x)-\eta_{t}^{n}\left(x+\frac{1}{n}\right)\right)^{2}
$$

We conclude that

$$
\lim _{n \rightarrow \infty}\left\{\left\langle\mathcal{W}^{n}(f)\right\rangle_{t}-\int_{0}^{t} \frac{4}{n} \sum_{x \in \mathbb{T}_{n}} c_{x}\left(\eta_{s}^{n}\right) \mathcal{Y}_{s}^{n}\left(f_{x}^{n}\right)^{2} d s\right\}=0
$$

in the sense that this difference is uniformly bounded by a deterministic sequence which goes to 0 as $n \rightarrow \infty$. Now we explain how to change $f_{x}^{n}(z)$ by $\partial_{2} f(z, x)$ in the sum above. From the inequality $a^{2}-b^{2}=(a-b)(a+b)$, plus the Cauchy-Schwarz inequality and the inequality $(a+b)^{2} \leq 2 a^{2}+2 b^{2}$ we obtain that

$$
\begin{aligned}
\mathbb{E}_{n}\left[\left|\mathcal{Y}_{t}^{n}\left(f_{1}\right)^{2}-\mathcal{Y}_{t}^{n}\left(f_{2}\right)^{2}\right|\right] \leq & \mathbb{E}_{n}\left[\left(\mathcal{Y}_{t}^{n}\left(f_{1}\right)-\mathcal{Y}_{t}^{n}\left(f_{2}\right)\right)^{2}\right]^{\frac{1}{2}} \times \\
& \times\left(2\left\{\mathbb{E}_{n}\left[\mathcal{Y}_{t}^{n}\left(f_{1}\right)^{2}\right]+\mathbb{E}_{n}\left[\mathcal{Y}_{t}^{n}\left(f_{2}\right)^{2}\right]\right)^{\frac{1}{2}}\right.
\end{aligned}
$$

Using the product structure of $\nu_{\frac{1}{2}}$, the right-hand side of this inequality is equal to

$$
\frac{1}{4}\left(\frac{1}{n} \sum_{x \in \mathbb{T}_{n}}\left(f_{1}(x)-f_{2}(x)\right)^{2}\right)^{\frac{1}{2}}\left(\frac{1}{n} \sum_{x \in \mathbb{T}_{n}}\left(f_{1}(x)^{2}+f_{2}(x)^{2}\right)\right)^{\frac{1}{2}} .
$$

Therefore, the application $f \mapsto \mathcal{Y}_{t}^{n}(f)^{2}$ from $C(\mathbb{T})$ to $L^{1}$ is uniformly continuous in $n$ and $t$. Let $g_{x}: \mathbb{T} \rightarrow \mathbb{R}$ be defined as $g_{x}(z)=\partial_{2} f(z, x)$. We see that $f_{x}^{n}$ converges uniformly to $g_{x}$, the convergence being uniform in $n$ and $x$. We conclude that

$$
\lim _{n \rightarrow \infty} \int_{0}^{t} \frac{4}{n} \sum_{x \in \mathbb{T}_{n}} c_{x}\left(\eta_{s}^{n}\right)\left(\mathcal{Y}_{s}^{n}\left(f_{x}^{n}\right)^{2}-\mathcal{Y}_{s}^{n}\left(g_{x}\right)^{2}\right) d s=0
$$

in $L^{1}$. Now we need to get rid of the term $c_{x}\left(\eta_{s}^{n}\right)$, which is not a function of $\mathcal{Y}_{s}^{n}$. The idea is to take advantage of the continuity of $\mathcal{Y}_{t}^{n}\left(g_{x}\right)$ with respect to $x$, in order to introduce an average of $c_{x}$ over some finite interval. Note that for any $i \in \mathbb{Z}$,

$$
\lim _{n \rightarrow \infty} \sup _{x \in \mathbb{T}_{n}} \sup _{z \in \mathbb{T}}\left|g_{x}(z)-g_{x}\left(z+\frac{i}{n}\right)\right|=0 .
$$

This together with (3.22) gives that for any $\ell \in \mathbb{N}$,

$$
\lim _{n \rightarrow \infty} \int_{0}^{t} \frac{4}{n} \sum_{x \in \mathbb{T}_{n}} c_{x}\left(\eta_{s}^{n}\right)\left(\mathcal{Y}_{s}^{n}\left(g_{x}\right)^{2}-\frac{1}{2 \ell+1} \sum_{i=-\ell}^{\ell} \mathcal{Y}_{s}^{n}\left(g_{x}\left(\cdot+\frac{i}{n}\right)\right)^{2}\right) d s=0
$$

in $L^{1}$. Passing the sum over $i$ to the term with $\eta$, we see that

$$
\lim _{n \rightarrow \infty}\left\{\left\langle\mathcal{W}^{n}(f)\right\rangle_{t}-\int_{0}^{t} \frac{4}{n} \sum_{x \in \mathbb{T}_{n}}\left(\frac{1}{2 \ell+1} \sum_{i=-\ell}^{\ell} c_{x+i}\left(\eta_{s}^{n}\right)\right) \mathcal{Y}_{s}^{n}\left(g_{x}\right)^{2} d s\right\}=0
$$

in $L^{1}$. Now, note that, with respect to $\nu_{\frac{1}{2}}$, the expectation of $c_{x}\left(\eta_{s}^{n}\right)$ with respect to $\nu_{1 / 2}$ is equal to $\frac{1}{2}$ and $c_{x}$ and $c_{y}$ are independent as soon as $|y-x|>\frac{1}{n}$. If we 
split the sum

$$
\frac{1}{2 \ell+1} \sum_{i=-\ell}^{\ell} c_{x+i}(\eta)
$$

into two sums, one running over even values of $i$ and another running over odd values of $i$, we can use the elementary inequality $|a+b|^{2 p} \leq 2^{p-1}\left(|a|^{2 p}+|b|^{2 p}\right)$ plus Burkholder's inequality on each sum to get the bound

$$
E_{\frac{1}{2}}\left[\left|\frac{1}{2 \ell+1} \sum_{i=-\ell}^{\ell} c_{x+i}(\eta)-\frac{1}{2}\right|^{2 p}\right] \leq \frac{C_{p}}{\ell^{p}}
$$

for any $p \geq 1$ and any $\ell \in \mathbb{N}$, where $C_{p}$ is a constant that depends only on $p$. In particular, using Hölder's inequality (to split the product) and Burkholder's inequality (for $\mathcal{Y}_{s}^{n}\left(g_{x}\right)$ ), we get the bound

$$
\mathbb{E}_{n}\left[\left|\frac{1}{2 \ell+1} \sum_{i=-\ell}^{\ell} c_{x+i}\left(\eta_{s}^{n}\right)-\frac{1}{2}\right| \mathcal{Y}_{s}^{n}\left(g_{x}\right)^{2}\right] \leq \frac{c_{3}(f)}{\sqrt{\ell}}
$$

for some constant $c_{3}(f)$ which does not depend on $n \in \mathbb{N}$ nor $s \in[0, T]$. We conclude that

$$
\varlimsup_{\ell \rightarrow \infty} \varlimsup_{n \rightarrow \infty} \int_{0}^{t} \frac{4}{n} \sum_{x \in \mathbb{T}_{n}} \frac{1}{2 \ell+1} \sum_{i=-\ell}^{\ell}\left(c_{x+i}\left(\eta_{s}^{n}\right)-\frac{1}{2}\right) \mathcal{Y}_{s}^{n}\left(g_{x}\right)^{2} d s=0
$$

in $L^{1}$. In particular,

$$
\lim _{n \rightarrow \infty}\left\{\left\langle\mathcal{W}^{n}(f)\right\rangle_{t}-2 \int_{0}^{t} \frac{1}{n} \sum_{x \in \mathbb{T}_{n}} \mathcal{Y}_{s}^{n}\left(g_{x}\right)^{2} d s\right\}=0
$$

in $L^{1}$. Both terms in this limit are convergent in distribution when $n \rightarrow \infty$.

3.5. The martingale problem. Putting Theorems 3.4 (actually Corollaries 3.6 and 3.7 ) and 3.8 together, we see that the process $\left\{Q_{t} ; t \in[0, T]\right\}$ satisfies the following martingale problem.

(MP) For any symmetric function $f \in C^{\infty}\left(\mathbb{T}^{2}\right)$, the process

$$
\mathcal{W}_{t}(f)=Q_{t}(f)-Q_{0}(f)-\int_{0}^{t} Q_{s}(\Delta f) d s
$$

is a continuous martingale of quadratic variation

$$
\langle\mathcal{W}(f)\rangle_{t}=\int_{0}^{t} \int_{\mathbb{T}}\left(\mathcal{Y}_{s}\left(\partial_{1} f(x, \cdot)\right)^{2}+\mathcal{Y}_{s}\left(\partial_{2} f(\cdot, x)\right)^{2}\right) d x d s .
$$

Formally speaking, this is the martingale problem associated with the stochastic partial differential equation

$$
d Q_{t}(x, y)=\Delta Q_{t}(x, y) d t+\frac{1}{\sqrt{2}}\left(\mathcal{Y}_{t}(x) \nabla \tilde{\omega}(y, t)+\mathcal{Y}_{t}(y) \nabla \tilde{\omega}(x, t)\right) d t,
$$

where $\tilde{\omega}$ is a space-time white noise in $\mathbb{T} \times[0, T]$. We note that this martingale problem does not tell us anything about the relation between the processes $\left\{\mathcal{Y}_{t} ; t \in\right.$ $[0, T]\}$ and $\tilde{\omega}$, but, in fact, we would like to say that $\tilde{\omega}$ is the white noise appearing 
in (2.7). Let us consider a test function of the form $f(x, y)=f_{1}(x) f_{2}(y)$ for some functions $f_{1}, f_{2} \in C^{\infty}(\mathbb{T})$. Then, by (3.21) we have

$$
\langle\mathcal{W}(f)\rangle_{t}=2\left\|\nabla f_{2}\right\|^{2} \int_{0}^{t} \mathcal{Y}_{s}\left(f_{1}\right)^{2} d s .
$$

In the other hand, by definition of $\left\{Q_{t}^{n} ; t \in[0, T]\right\}$, we see that

$$
Q_{t}^{n}(f)=\mathcal{Y}_{t}^{n}\left(f_{1}\right) \mathcal{Y}_{t}^{n}\left(f_{2}\right)-\frac{1}{4 n} \sum_{x \in \mathbb{T}_{n}} f_{1}(x) f_{2}(x),
$$

and taking the limit along the subsequence $n$, we conclude that

$$
Q_{t}(f)=\mathcal{Y}_{t}\left(f_{1}\right) \mathcal{Y}_{t}\left(f_{2}\right)-\frac{1}{4} \int_{\mathbb{T}} f_{1}(x) f_{2}(x) d x .
$$

From Itô's formula, we can obtain the martingale decomposition of the process $\left\{\mathcal{Y}_{t}\left(f_{1}\right) \mathcal{Y}_{t}\left(f_{2}\right) ; t \in[0, T]\right\}$. In particular, writing $d \omega_{t}(f)$ as the Itô differential of the martingale process $\int_{0}^{t} \int_{\mathbb{T}} f(x) \omega(d x d s)$,

$$
\begin{aligned}
\mathcal{Y}_{t}\left(f_{1}\right) \mathcal{Y}_{t}\left(f_{2}\right)=\mathcal{Y}_{0}\left(f_{1}\right) \mathcal{Y}_{0}\left(f_{2}\right) & +\int_{0}^{t}\left\{\mathcal{Y}_{s}\left(\Delta f_{1}\right) \mathcal{Y}_{s}\left(f_{2}\right)+\mathcal{Y}_{s}\left(f_{1}\right) \mathcal{Y}_{s}\left(\Delta f_{2}\right)\right\} d s \\
& +\frac{1}{\sqrt{2}} \int_{0}^{t}\left\{\mathcal{Y}_{s}\left(f_{1}\right) d \omega_{s}\left(\nabla f_{2}\right)+\mathcal{Y}_{s}\left(f_{2}\right) d \omega_{s}\left(\nabla f_{1}\right)\right\} \\
& +\frac{t}{2} \int_{\mathbb{T}} \nabla f_{1}(x) \nabla f_{2}(x) d x .
\end{aligned}
$$

Let us rewrite this identity in terms of the process $\left\{Q_{t} ; t \in[0, T]\right\}$. Now note that $\Delta f=f_{1} \Delta f_{2}+f_{2} \Delta f_{1}$ and, by an integration by parts, it holds that

$$
\int_{0}^{t} Q_{s}(\Delta f) d s=\int_{0}^{t}\left\{\mathcal{Y}_{s}\left(\Delta f_{1}\right) \mathcal{Y}_{s}\left(f_{2}\right)+\mathcal{Y}_{s}\left(f_{1}\right) \mathcal{Y}_{s}\left(\Delta f_{2}\right)\right\} d s+\frac{t}{2} \int_{\mathbb{T}} \nabla f_{1}(x) \nabla f_{2}(x) d x .
$$

From this we conclude that

$$
Q_{t}(f)=Q_{0}(f)+\int_{0}^{t} Q_{s}(\Delta f) d s+\frac{1}{\sqrt{2}} \int_{0}^{t}\left\{\mathcal{Y}_{s}\left(f_{1}\right) d \omega_{s}\left(\nabla f_{2}\right)+\mathcal{Y}_{s}\left(f_{2}\right) d \omega_{s}\left(\nabla f_{1}\right)\right\}
$$

for any function $f$ of the form $f(x, y)=f_{1}(x) f_{2}(y)$. Therefore, at least for functions of this product form, we are able to identify $\tilde{\omega}$ with $\omega$. Actually, since the set of linear combinations of functions of the form $f_{1}(x) f_{2}(y)$ is dense in $C^{\infty}\left(\mathbb{T}^{2}\right)$, this relation allows us to identify the martingale appearing in (MP) in terms of $\omega$. Let us explain this in a more rigorous way. Let us define the $D^{\prime}(\mathbb{T})$-valued process $\left\{\mathcal{N}_{t} ; t \in[0, T]\right\}$ as

$$
\mathcal{N}_{t}(f)=\mathcal{Y}_{t}(f)-\mathcal{Y}_{0}(f)-\int_{0}^{t} \mathcal{Y}_{s}(\Delta f) d s
$$

for any $f \in C^{\infty}(\mathbb{T})$. By the definition of $\left\{\mathcal{Y}_{t} ; t \in[0, T]\right\}$, the process $\left\{\mathcal{N}_{t}(f) ; t \in\right.$ $[0, T]\}$ is a continuous martingale of quadratic variation

$$
\langle\mathcal{N}(f)\rangle_{t}=\frac{t}{2} \int_{\mathbb{T}}(\nabla f(x))^{2} d x .
$$

The relation (3.33) can be rewritten as

$$
\mathcal{W}_{t}(f)=\frac{1}{\sqrt{2}} \int_{0}^{t}\left\{\mathcal{Y}_{s}\left(f_{1}\right) d \mathcal{N}_{s}\left(f_{2}\right)+\mathcal{Y}_{s}\left(f_{2}\right) d \mathcal{N}_{s}\left(f_{1}\right)\right\}
$$


Note that a by-product of our proof of tightness for $\left\{Q_{t}^{n} ; t \in[0, T]\right\}_{n \in \mathbb{N}}$ is a proof of tightness for $\left\{\mathcal{W}_{t}^{n} ; t \in[0, T]\right\}$ as a $D^{\prime}\left(\mathbb{T}^{2}\right)$-valued martingale. Therefore, $\left\{\mathcal{W}_{t} ; t \in[0, T]\right\}$ is a well-defined, $D^{\prime}\left(\mathbb{T}^{2}\right)$-valued martingale process. In particular, its distribution is determined by the values of $\left\{\mathcal{W}_{t}(f) ; t \in[0, T]\right\}$ for $f$ of the form $f_{1}(x) f_{2}(y)$. In (3.35) there is no mention to the process $\left\{Q_{t} ; t \in[0, T]\right\}$. We are finally ready to establish a uniqueness result for the martingale problem (MP). We state it as a theorem.

Theorem 3.9. Let $\left\{\left(Q_{t}, \mathcal{Y}_{t}, \mathcal{N}_{t}\right) ; t \in[0, T]\right\}$ be a $D^{\prime}\left(\mathbb{T}^{2}\right) \otimes D^{\prime}(\mathbb{T}) \otimes D^{\prime}(\mathbb{T})$-valued, continuous processes. Assume that

a) for any $f \in C^{\infty}(\mathbb{T})$, the process $\left\{\mathcal{N}_{t}(f) ; t \in[0, T]\right\}$ is a continuous martingale of quadratic variation $\frac{1}{2} t \int_{\mathbb{T}}(\nabla f(x))^{2} d x$,

b) for any $f \in C^{\infty}(\mathbb{T})$, the process $\left\{\mathcal{Y}_{t}(f) ; t \in[0, T]\right\}$ satisfies the relation

$$
\mathcal{Y}_{t}(f)=\mathcal{Y}_{0}(f)+\int_{0}^{t} \mathcal{Y}_{s}(\Delta f) d s+\mathcal{N}_{t}(f),
$$

c) there exists a $\mathcal{D}\left(\mathbb{T}^{2}\right)$-valued process $\left\{\mathcal{W}_{t} ; t \in[0, T]\right\}$ such that for any $f, g \in$ $C^{\infty}(\mathbb{T})$

$$
\mathcal{W}_{t}(f(x) g(y))=\frac{1}{\sqrt{2}} \int_{0}^{t}\left\{\mathcal{Y}_{s}(f) d \mathcal{N}_{s}(g)+\mathcal{Y}_{s}(g) d \mathcal{N}_{s}(f)\right\},
$$

d) for any $f \in C^{\infty}\left(\mathbb{T}^{2}\right)$, we have

$$
Q_{t}(f)=Q_{0}(f)+\int_{0}^{t} Q_{s}(\Delta f) d s+\mathcal{W}_{t}(f),
$$

e) for any $f \in C^{\infty}\left(\mathbb{T}^{2}\right)$ and any $t \in[0, T]$, the real-valued random variable $Q_{t}(f)$ has a Gaussian distribution of mean zero and variance $\frac{1}{4} \iint_{\mathbb{T}^{2}} f(x, y)^{2} d x d y$.

Then the distribution of $\left\{\left(Q_{t}, \mathcal{Y}_{t}, \mathcal{N}_{t}\right) ; t \in[0, T]\right\}$ is uniquely determined.

Proof: The proof was basically accomplished above. Note that the tightness result shows existence of the triple $\left\{\left(Q_{t}, \mathcal{Y}_{t}, \mathcal{N}_{t}\right) ; t \in[0, T]\right\}$. Condition e) implies stationarity of the process $\left\{Q_{t} ; t \in[0, T]\right\}$, and could be relaxed to some moment bound, but since we are only interested on the stationary case, we will not discuss these generalizations here. Using this stationarity condition, it is not hard to show that for any $t \in[0, T]$ and any smooth path $F:[0, t] \rightarrow C^{\infty}\left(\mathbb{T}^{2}\right)$,

$$
Q_{t}\left(F_{t}\right)=Q_{0}\left(F_{0}\right)+\int_{0}^{t} Q_{s}\left(\left(\partial_{s}+\Delta\right) F_{s}\right) d s+\int_{0}^{t} d \mathcal{W}_{s}\left(F_{s}\right) .
$$

This relation allows us to use Duhamel's formula to compute $Q_{t}(f)$ in terms of the process $\left\{\mathcal{W}_{t} ; t \in[0, T]\right\}$. Fix $f \in C^{\infty}\left(\mathbb{T}^{2}\right)$ and $t \in[0, T]$, and consider the test function $F_{s}=P_{t-s} f$, where $\left\{P_{t} ; t \in[0, T]\right\}$ is the semigroup generated by $\Delta$. Then, $\left(\partial_{s}+\Delta\right) F_{s}=0$ and

$$
Q_{t}(f)=Q_{0}\left(P_{t} f\right)+\int_{0}^{t} d \mathcal{W}_{s}\left(P_{t-s} f\right) .
$$

Therefore, given the process $\left\{\mathcal{W}_{t} ; t \in[0, T]\right\}$ and the initial distribution of $Q_{0}$, the process $\left\{Q_{t} ; t \in[0, T]\right\}$ is uniquely determined by this Duhamel's formula, which shows uniqueness in distribution. 
We finish this section with a note on the proof of Theorem 2.4. Recall that in Section 3 we showed tightness of the sequence of processes $\left\{Q_{t}^{n} ; t \in[0, T]\right\}_{n \in \mathbb{N}}$. In Section 3.4 we proved that any limit point $\left\{Q_{t} ; t \in[0, T]\right\}$ of the sequence $\left\{Q_{t}^{n} ; t \in[0, T]\right\}_{n \in \mathbb{N}}$ satisfies the martingale problem stated in Theorem 3.9, and then in Section 3.5 we proved that this martingale problem has a unique solution in distribution. From all this, we conclude that the limit point of $\left\{Q_{t}^{n} ; t \in[0, T]\right\}$ is unique, and as a consequence we conclude that the whole sequence converges to that limit point.

\section{Properties of the quadratic fluctuation field at the diagonal}

In this section we prove Theorems 2.5, 2.7 and 2.8. The idea is to use the martingale characterization of the process $\left\{Q_{t} ; t \in[0, T]\right\}$ obtained in Theorem 3.9, and more precisely the martingale decomposition (3.38) in order to rewrite the integral process

$$
\int_{0}^{t} Q_{s}\left(\left(f^{\prime} \otimes \delta\right) * \iota_{\varepsilon}\right) d s
$$

as a martingale plus a function of $Q_{t}$ and $Q_{0}$. For a given function $g \in C^{\infty}\left(\mathbb{T}^{2}\right)$, let $\psi_{g}: \mathbb{T}^{2} \rightarrow \mathbb{R}$ denote the solution of the Poisson equation

$$
\Delta \psi=g .
$$

If $\iint_{\mathbb{T}^{2}} g(x, y) d x d y=0$, then the solution $\psi_{g}$ of this equation belongs to $C^{\infty}\left(\mathbb{T}^{2}\right)$, and by (3.38) we have that

$$
\int_{0}^{t} Q_{s}(g) d s=Q_{t}\left(\psi_{g}\right)-Q_{0}\left(\psi_{g}\right)-\mathcal{W}_{t}\left(\psi_{g}\right)
$$

4.1. Proof of Theorem 2.5. In order to prove the theorem we start by showing that for any $t \in[0, T]$ and any $f \in C^{\infty}(\mathbb{T})$, the sequence $\left\{\mathcal{A}_{t}^{\varepsilon}(f)\right\}_{\varepsilon}$ is Cauchy in $L^{2}(P)$, and therefore the limit

$$
\mathcal{A}_{t}(f)=\lim _{\varepsilon \rightarrow 0} \mathcal{A}_{t}^{\varepsilon}(f)
$$

exists in $L^{2}(P)$. To prove that $\left\{\mathcal{A}_{t}^{\varepsilon}(f)\right\}_{\varepsilon}$ is a Cauchy sequence, we note that

$$
\mathcal{A}_{t}^{\varepsilon}(f)=Q_{t}\left(\psi_{f}^{\varepsilon}\right)-Q_{0}\left(\psi_{f}^{\varepsilon}\right)-\mathcal{W}_{t}\left(\psi_{f}^{\varepsilon}\right),
$$

where $\psi_{f}^{\varepsilon}$ is the solution of the Poisson equation (4.2) with $g=\left(f^{\prime} \otimes \delta\right) * \iota_{\varepsilon}$ and satisfying $\int_{\mathbb{T}} \psi_{f}^{\varepsilon}(x) d x=0$. Therefore, from the inequality $(x+y)^{2} \leq 2 x^{2}+2 y^{2}$, we have, for $0<\delta<\varepsilon$, that

$$
\begin{aligned}
E\left[\left(\mathcal{A}_{t}^{\varepsilon}(f)-\mathcal{A}_{t}^{\delta}(f)\right)^{2}\right] \leq & 2 E\left[\left(Q_{t}\left(\psi_{f}^{\varepsilon}-\psi_{f}^{\delta}\right)-Q_{0}\left(\psi_{f}^{\varepsilon}-\psi_{f}^{\delta}\right)\right)^{2}\right] \\
& +2 E\left[\mathcal{W}_{t}\left(\psi_{f}^{\varepsilon}-\psi_{f}^{\delta}\right)^{2}\right] .
\end{aligned}
$$

Recall that variance of the martingale $\mathcal{W}_{t}\left(\psi_{f}^{\varepsilon}-\psi_{f}^{\delta}\right)$ is equal to $\frac{1}{2} t\left\|\nabla\left(\psi_{f}^{\varepsilon}-\psi_{f}^{\delta}\right)\right\|^{2}$. In the first lemma below, we prove that the second expectation on the right-hand side of last expression is bounded by $C t \varepsilon\left\|f^{\prime}\right\|^{2}$. On the second lemma below we prove that the first expectation on the right-hand side of last expression is also bounded by $C t \varepsilon\left\|f^{\prime}\right\|^{2}$. From those results, it follows that

$$
E\left[\left(\mathcal{A}_{t}^{\varepsilon}(f)-\mathcal{A}_{t}^{\delta}(f)\right)^{2}\right] \leq C t \varepsilon\left\|f^{\prime}\right\|^{2}
$$

and we are done. 
Lemma 4.1. For $\psi_{f}^{\varepsilon}$ given above, there exists a constant $C$ such that

$$
\left\|\nabla\left(\psi_{f}^{\varepsilon}-\psi_{f}^{\delta}\right)\right\|^{2} \leq C \varepsilon\left\|f^{\prime}\right\|^{2}
$$

for any $0<\delta<\varepsilon<1$ and any $f \in C^{\infty}(\mathbb{T})$.

Proof: We will obtain an explicit expression for the Fourier series of $\psi_{f}^{\varepsilon}$. For that purpose, for $k, m \in \mathbb{Z}$, let $\psi_{k, m}: \mathbb{T}^{2} \rightarrow \mathbb{C}$ be the trigonometric polynomial given by $\psi_{k, m}(x, y)=e^{2 \pi i(k x+m y)}$. Then $\left\{\psi_{k, m} ; k, m \in \mathbb{Z}\right\}$ is an orthonormal basis of $L^{2}\left(\mathbb{T}^{2}\right)$. For any function $g \in L^{2}\left(\mathbb{T}^{2}\right)$, we denote by $\widehat{g}: \mathbb{Z}^{2} \rightarrow \mathbb{C}$ the Fourier series of $g$ :

$$
\widehat{g}(k, m)=\iint_{\mathbb{T}^{2}} g(x, y) \bar{\psi}_{k, m}(x, y) d x d y
$$

for any $k, m \in \mathbb{Z}$. Then, a simple computation shows that

$$
\widehat{\Delta g}(k, m)=-4 \pi^{2}\left(k^{2}+m^{2}\right) \widehat{g}(k, m),
$$

where $\Delta g$ denotes the two-dimensional laplacian of $g$. Also note that

$$
\left(f^{\prime} \widehat{\otimes \delta}\right) * \iota_{\varepsilon}(k, m)=\left(\widehat{f^{\prime} \otimes \delta}\right)(k, m) \widehat{\iota_{\varepsilon}}(k, m)=2 \pi i(k+m) \widehat{f}(k+m) \widehat{\iota_{\varepsilon}}(k, m),
$$

from where we get that

$$
\widehat{\psi}_{f}^{\varepsilon}(k, m)=\frac{-i(k+m) \widehat{f}(k+m) \widehat{\iota}_{\varepsilon}(k, m)}{2 \pi\left(k^{2}+m^{2}\right)} .
$$

The Fourier series $\widehat{\iota}_{\varepsilon}(k, m)$ can be computed explicitly. In fact,

$$
\widehat{\iota}_{\varepsilon}(k, m)=\frac{\sin (2 \pi k \varepsilon) \sin (2 \pi m \varepsilon)}{4 \pi^{2} \varepsilon^{2} k m},
$$

where we use the convention $\frac{\sin (x)}{x}=1$ for $x=0$. By Parseval's identity, for any function $\psi \in L^{2}\left(\mathbb{T}^{2}\right)$,

$$
\|\psi\|^{2}=\sum_{k, m \in \mathbb{Z}}|\widehat{\psi}(k, m)|^{2}
$$

We claim that there exists a universal constant $C>0$ such that

$$
\left|\widehat{\iota}_{\varepsilon}(k, m)-\widehat{\iota}_{\delta}(k, m)\right| \leq C(\varepsilon-\delta)\left(k^{2}+m^{2}\right)^{1 / 2}
$$

for any $0<\delta \leq \varepsilon$. In fact, it is enough to note that the gradient of the function $(x, y) \mapsto \frac{\sin (x) \sin (y)}{x y}$ is bounded in $\mathbb{R}^{2}$. Since the function $x \mapsto \frac{\sin (x)}{x}$ is bounded between -1 and 1 , we also have the trivial bound

$$
\left|\widehat{\iota}_{\varepsilon}(k, m)-\widehat{\iota}_{\delta}(k, m)\right| \leq 2,
$$

which is actually better than (4.9) if $\left(k^{2}+m^{2}\right)^{1 / 2} \geq \frac{2}{C(\varepsilon-\delta)}$. From Parseval's identity and from the inequality $(k+m)^{2} \leq 2\left(k^{2}+m^{2}\right)$ we get that

$$
\left\|\nabla\left(\psi_{f}^{\varepsilon}-\psi_{f}^{\delta}\right)\right\|^{2} \leq 2 \sum_{k, m \in \mathbb{Z}} \frac{(k+m)^{2}|\widehat{f}(k+m)|^{2}}{k^{2}+m^{2}}\left|\widehat{\iota}_{\varepsilon}(k, m)-\widehat{\iota}_{\delta}(k, m)\right|^{2} .
$$

We split the sum above into a sum over two sets, depending on whether (4.9) or (4.10) is the best estimate. We start with the case on which the trivial bound (4.10) is the best one. Note at first that the set

$$
R_{1}=\left\{(k, m) \in \mathbb{Z}^{2} ;\left(k^{2}+m^{2}\right)^{1 / 2} \geq \frac{2}{C(\varepsilon-\delta)}\right\}
$$


is contained on the set

$$
R_{1}^{\prime}=\left\{(k, m) \in \mathbb{Z}^{2} ;|k+m| \geq \frac{2}{C(\varepsilon-\delta)},|k-m| \geq \frac{2}{C(\varepsilon-\delta)}\right\} .
$$

Let us define the new coordinates $\ell=k+m, n=k-m$. In these new coordinates, the set $R_{1}^{\prime}$ is given by

$$
R_{1}^{\prime}=\left\{|\ell| \geq \frac{2}{C(\varepsilon-\delta)},|n| \geq \frac{2}{C(\varepsilon-\delta)}\right\} .
$$

Using the estimate $k^{2}+m^{2} \geq \frac{1}{2} n^{2}$, we have that there exists a constant $C_{1}$ such that for any $\varepsilon$ small enough, it holds that

$$
\begin{aligned}
& \sum_{(k, m) \in R_{1}^{\prime}} \frac{(k+m)^{2}|\widehat{f}(k+m)|^{2}}{k^{2}+m^{2}}\left|\widehat{\iota}_{\varepsilon}(k, m)-\widehat{\iota}_{\delta}(k, m)\right|^{2} \\
& \leq 8 \sum_{(\ell, n) \in R_{1}^{\prime}} \frac{\ell^{2}}{n^{2}}|\widehat{f}(\ell)|^{2} \leq C_{1}(\varepsilon-\delta)\left\|f^{\prime}\right\|^{2},
\end{aligned}
$$

Now we estimate the sum in (4.11) over the set $\left(R_{1}^{\prime}\right)^{c}$. Using (4.9), we see that

$$
\begin{aligned}
\sum_{(k, m) \in\left(R_{1}^{\prime}\right)^{c}} \frac{(k+m)^{2}|\widehat{f}(k+m)|^{2}}{k^{2}+m^{2}}\left|\widehat{\iota}_{\varepsilon}(k, m)-\widehat{\iota}_{\delta}(k, m)\right|^{2} & \leq C^{2}(\varepsilon-\delta)^{2} \sum_{(\ell, n) \in\left(R_{1}^{\prime}\right)^{c}} \ell^{2}|\widehat{f}(\ell)|^{2} \\
& \leq 2 C^{3}(\varepsilon-\delta)\left\|f^{\prime}\right\|^{2} .
\end{aligned}
$$

This together with the previous estimate ends the proof of the lemma.

Remark 4.2. At first glance, it seems that solving the Poisson equation (4.2) with $f^{\prime}$ instead of $f$ is not needed in order to prove this lemma, since the final bound depends only on $f^{\prime}$ and not on $f$. Actually, what we need is the integral condition $\int_{\mathbb{T}} f^{\prime}(x) d x=0$, which is equivalent to $f^{\prime}$ being the actual derivative of a function $f$. This condition means that the 0 -th order of the Fourier's expansion of $f^{\prime}$ vanishes, allowing to divide by $k^{2}+m^{2}$ without worrying about the case $k=0, m=0$ in various steps of the computations.

Lemma 4.3. For any $g \in L^{2}\left(\mathbb{T}^{2}\right)$,

$$
E\left[\left(Q_{t}(g)-Q_{0}(g)\right)^{2}\right]=\frac{1}{2}\left\langle g, g-P_{t} g\right\rangle \leq t\|\nabla g\|^{2} .
$$

Proof: Let us assume that $g \in C^{\infty}\left(\mathbb{T}^{2}\right)$. By Duhamel's formula (3.40), we have that

$$
\begin{aligned}
E\left[\left(Q_{t}(g)-Q_{0}(g)\right)^{2}\right] & =E\left[Q_{0}\left(P_{t} g-g\right)^{2}\right]+E\left[\left(\int_{0}^{t} d \mathcal{W}_{s}\left(P_{t-s} g\right)\right)^{2}\right] \\
& =\frac{1}{4}\left\|P_{t} g-g\right\|^{2}+\frac{1}{2} \int_{0}^{t}\left\|\nabla\left(P_{s} g\right)\right\|^{2} d s,
\end{aligned}
$$

where $P_{t}$ is the semigroup generated by $\Delta$. Since

$$
\nabla\left(P_{s} g\right)\left\|^{2}=\frac{1}{2} \frac{d}{d t}\right\| P_{t} g \|^{2}
$$

the lemma follows for $g \in C^{\infty}\left(\mathbb{T}^{2}\right)$. Since $Q_{t}$ and $P_{t}$ are continuous under approximations in $L^{2}\left(\mathbb{T}^{2}\right)$, the lemma follows for $g \in L^{2}\left(\mathbb{T}^{2}\right)$ by approximations. Now, note that

$$
\frac{d}{d t}\left\langle g, g-P_{t} g\right\rangle=\left\|\nabla P_{t} g\right\|^{2} \leq\|\nabla g\|^{2}
$$


and in particular $\left\langle g, g-P_{t} g\right\rangle \leq t\|\nabla g\|^{2}$.

Recall that for each fixed time $t, Q_{t}$ is a white noise of variance $\frac{1}{4}$. Therefore,

$$
E\left[Q_{t}\left(\psi_{f}^{\varepsilon}\right)^{2}\right]=\frac{1}{4}\left\|\psi_{f}^{\varepsilon}\right\|^{2}
$$

for any $t \in[0, T]$. By using the representation

$$
\mathcal{A}_{t}^{\varepsilon}(f)=\int_{0}^{t} Q_{s}\left(\left(f^{\prime} \otimes \delta\right) * \iota_{\varepsilon}\right) d s,
$$

and the Cauchy-Schwarz's inequality, we get the simple bound

$$
E\left[\mathcal{A}_{t}^{\varepsilon}(f)^{2}\right] \leq \frac{1}{4} t^{2}\left\|\left(f^{\prime} \otimes \delta\right) * \iota_{\varepsilon}\right\|^{2} \leq \frac{C t^{2}}{\varepsilon}\left\|f^{\prime}\right\|^{2} .
$$

Taking $\delta \rightarrow 0$, choosing $\varepsilon=\sqrt{t}$ and combining (4.7) with (4.16), we obtain the bound

$$
E\left[\mathcal{A}_{t}(f)^{2}\right] \leq C t^{3 / 2}\left\|f^{\prime}\right\|^{2} .
$$

These estimates are all we need in order to show that $\left\{\mathcal{A}_{t} ; t \in[0, T]\right\}$ is a welldefined, $D^{\prime}\left(\mathbb{T}^{2}\right)$-valued process and to show that $\left\{\mathcal{A}_{t}^{\varepsilon} ; t \in[0, T]\right\}$ converges to $\left\{\mathcal{A}_{t} ; t \in[0, T]\right\}$ at the level of processes, see Theorem 2.1 of Gonçalves and Jara (2013) for the details.

4.2. Proof of Theorem 2.7: small-time asymptotics of the quadratic field. Let us denote by $\psi_{f}$ the solution of the Poisson equation (4.2) with $g=f^{\prime} \otimes \delta$ satisfying $\int_{\mathbb{T}^{2}} \psi_{f}(x) d x=0$. By Lemma $4.1, \psi \in \mathcal{H}_{1,2}\left(\mathbb{T}^{2}\right)$. Recall from the previous section that, for any $t \in[0, T]$, we have the following expression for $\mathcal{A}_{t}(f)$ :

$$
\mathcal{A}_{t}(f)=Q_{0}\left(P_{t} \psi_{f}-\psi_{f}\right)+\int_{0}^{t} d \mathcal{W}_{s}\left(P_{t-s} \psi_{f}-\psi_{f}\right) .
$$

The variance of the first term on the right-hand side of (4.18) is equal to $\frac{1}{4} \| P_{t} \psi_{f}-$ $\psi_{f} \|^{2}$, while the variance of the second term on the right-hand side of (4.18) is equal to

$$
\frac{1}{2} \int_{0}^{t}\left\|P_{t-s} \psi_{f}-\psi_{f}\right\|^{2} d s=\frac{1}{2} \int_{0}^{t}\left\|P_{s} \psi_{f}-\psi_{f}\right\|^{2} d s .
$$

In the next lemma we prove that the norm $\left\|P_{t} \psi_{f}-\psi_{f}\right\|$ decays to 0 like $t^{3 / 2}$.

Lemma 4.4. For any smooth function $f: \mathbb{T} \rightarrow \mathbb{R}$ we have that

$$
\lim _{t \rightarrow 0} \frac{\left\|P_{t} \psi_{f}-\psi_{f}\right\|^{2}}{t^{3 / 2}}=\frac{4}{3 \sqrt{\pi}}(-1+\sqrt{2}) \int_{\mathbb{T}} f(x)(-\Delta f)(x) d x .
$$

Proof: Note that for any $\psi: \mathbb{T}^{2} \rightarrow \mathbb{R}$ regular enough

$$
\widehat{P_{t} \psi}(k, m)=e^{-4 \pi^{2}\left(k^{2}+m^{2}\right) t} \widehat{\psi}(k, m) .
$$

Therefore, by Parseval's identity we have that

$$
\left\|P_{t} \psi_{f}-\psi_{f}\right\|^{2}=\sum_{k, m \in \mathbb{Z}} \frac{(k+m)^{2}|\widehat{f}(k+m)|^{2}}{4 \pi^{2}\left(k^{2}+m^{2}\right)^{2}}\left(1-e^{-4 \pi^{2}\left(k^{2}+m^{2}\right) t}\right)^{2} .
$$

From the change of variables $u=k+m, v=k-m$ we obtain that

$$
\left\|P_{t} \psi_{f}-\psi_{f}\right\|^{2}=\sum_{u, v} \frac{u^{2}|\widehat{f}(u)|^{2}}{\pi^{2}\left(u^{2}+v^{2}\right)^{2}}\left(1-e^{-\pi^{2}\left(u^{2}+v^{2}\right) t}\right)^{2},
$$


where the sum is over $u, v \in \mathbb{Z}$ such that $u+v$ is even. Last identity can be rewritten as

$$
\frac{\left\|P_{t} \psi_{f}-\psi_{f}\right\|^{2}}{t^{3 / 2}}=\sum_{u \in \mathbb{Z}} \frac{u^{2}|\widehat{f}(u)|^{2}}{2 \pi^{2}}\left(2 \sqrt{t} \sum_{\substack{v \in \mathbb{Z} \\ u+v \text { even }}} g(u \sqrt{t}, v \sqrt{t})^{2}\right) .
$$

where $g: \mathbb{R}^{2} \rightarrow \mathbb{R}$ is given by

$$
g(x, y)=\frac{1-e^{-\pi^{2}\left(x^{2}+y^{2}\right)}}{x^{2}+y^{2}} .
$$

Therefore, to finish the proof, it is enough to find the limit of the term at the right hand side of last expression. Note that the sum between parentheses is a Riemann sum for the integral

$$
\int_{\mathbb{R}} g(u \sqrt{t}, y)^{2} d y
$$

where the factor 2 in front of the sum comes from the fact that the sum is over one half of the integers. Moreover, we note that there exists a finite constant $c$ such that

$$
|g(x, y)| \leq \frac{c}{1+y^{2}}, \quad\left|\partial_{y} g(x, y)\right| \leq \frac{c}{1+y^{4}}
$$

for any $x \in \mathbb{R}$. Therefore,

$$
\lim _{t \rightarrow 0} 2 \sqrt{t} \sum_{\substack{v \in \mathbb{Z} \\ u+v \text { even }}} g(x, v \sqrt{t})^{2}=\int_{\mathbb{R}} g(x, y)^{2} d y,
$$

uniformly in $x$ and, in particular, we have, for any $u \in \mathbb{Z}$, that

$$
\lim _{t \rightarrow 0} 2 \sqrt{t} \sum_{\substack{v \in \mathbb{Z} \\ u+v \text { even }}} g(u \sqrt{t}, v \sqrt{t})^{2}=\int_{\mathbb{R}} g(0, y)^{2} d y=\frac{8}{3} \pi^{7 / 2}(-1+\sqrt{2}) .
$$

From (4.24) we can exchange the limit and the summation to obtain

$$
\begin{aligned}
\lim _{t \rightarrow 0} \frac{\left\|P_{t} \psi_{f}-\psi_{f}\right\|^{2}}{t^{3 / 2}} & =\frac{4}{3} \pi^{3 / 2}(-1+\sqrt{2}) \sum_{u \in \mathbb{Z}} u^{2}|\widehat{f}(u)|^{2} \\
& =\frac{4}{3 \sqrt{\pi}}(-1+\sqrt{2}) \int_{\mathbb{T}} f(x)(-\Delta f)(x) d x,
\end{aligned}
$$

which proves the lemma.

Now, since $\left\|P_{\varepsilon t} \psi_{f}-\psi_{f}\right\|$ decays to 0 as $\varepsilon^{3 / 2}$, the second term on the right-hand side of (4.18) is of smaller order than the first term. In other words,

$$
\lim _{\varepsilon \rightarrow 0} \varepsilon^{-3 / 4} \mathcal{A}_{\varepsilon t}(f)=\lim _{\varepsilon \rightarrow 0} \varepsilon^{-3 / 4} Q_{0}\left(P_{\varepsilon t} \psi_{f}-\psi_{f}\right),
$$

whenever one of the limits exists. The process $Q_{0}$ is Gaussian and centered, and therefore it is easy to check the convergence ot the term above. In fact, this is exactly what Lemma 4.4 shows. We conclude that $\varepsilon^{-3 / 4} \mathcal{A}_{\varepsilon t}(f)$ converges in distribution as $\varepsilon \rightarrow 0$ to a Gaussian random variable of mean zero and variance

$$
\frac{4}{3 \sqrt{\pi}}(-1+\sqrt{2}) t^{3 / 2} \int_{\mathbb{T}} f(x)(-\Delta f)(x) d x .
$$


Since the process $\left\{\mathcal{A}_{t}(f) ; t \geq 0\right\}$ has stationary increments, we have proved convergence in the sense of finite-dimensional distributions. In order to get convergence at the level of processes, we only need to show tightness of the sequence $\left\{\varepsilon^{-3 / 4} \mathcal{A}_{\varepsilon t}(f) ; t \geq 0\right\}$. But this follows from the comment after (4.17).

Remark 4.5. Note that the process $\left\{\mathcal{B}_{t}(f) ; t \geq 0\right\}$ is a fractional Brownian motion of Hurst exponent $\frac{3}{4}$ which coincides with the process arising as the scaling limit of occupation times of the exclusion process Gonçalves and Jara (2013). This fact is a quantitative version of the formal claim which states that fluctuations of additive functionals of local functions of degree 1 (as, for example, the occupation time) are of the same nature as the fluctuations of additive functionals of extensive functions of degree 2 (as, for example, the quadratic field).

4.3. Proof of Theorem 2.8: large-time variance of the quadratic field. In the previous subsection we have seen how we can extract non-trivial information about the quadratic field $\left\{\mathcal{A}_{t} ; t \geq 0\right\}$ for small times using the representation (4.18). In this subsection we will see what can we say about the variance of $\mathcal{A}_{t}(f)$ when $t$ is large. Actually, it will be easier to work with the representation

$$
\mathcal{A}_{t}(f)=Q_{t}\left(\psi_{f}\right)-Q_{0}\left(\psi_{f}\right)-\mathcal{W}_{t}\left(\psi_{f}\right) .
$$

Last identity can be obtained by passing to the limit in $\varepsilon \rightarrow 0$ in (4.5). Recall that the variance of $Q_{t}\left(\psi_{f}\right)$ (and also of $Q_{0}\left(\psi_{f}\right)$ ) is equal to $\left\|\psi_{f}\right\|^{2}$, while the variance of $\mathcal{W}_{t}\left(\psi_{f}\right)$ is equal to $\frac{1}{2} t\left\|\nabla \psi_{f}\right\|^{2}$. Therefore, we see that

$$
\lim _{t \rightarrow \infty} \frac{E\left[\mathcal{A}_{t}(f)^{2}\right]}{t}=\frac{1}{2}\left\|\nabla \psi_{f}\right\|^{2} .
$$

The following lemma tells us the behavior of $\left\|\nabla \psi_{f}\right\|^{2}$ :

Lemma 4.6. There exists a bounded function $a: \mathbb{Z} \rightarrow \mathbb{R}$ such that

$$
\left\|\nabla \psi_{f}\right\|^{2}=\sum_{k \in \mathbb{Z}}\left(\pi|k|+\frac{a(k)}{|k|}\right)|\widehat{f}(k)|^{2} .
$$

Proof: A simple computation shows that

$$
\left\|\nabla \psi_{f}\right\|^{2}=\sum_{k, m} \frac{(k+m)^{4}|\widehat{f}(k+m)|^{2}}{\left(k^{2}+m^{2}\right)^{2}} .
$$

Using the change of variables $u=k+m, v=k-m$, we can rewrite this expression as

$$
\sum_{\substack{u \in \mathbb{Z} \\ u+v}} \sum_{\substack{v \in \mathbb{Z} \\ \text { is even }}} \frac{4|u|^{4}|\widehat{f}(u)|^{2}}{\left(u^{2}+v^{2}\right)^{2}}=\sum_{u \in \mathbb{Z}} 4|u||\widehat{f}(u)|^{2}\left(\frac{1}{|u|} \sum_{\substack{v \in \mathbb{Z} \\ v \text { is even }}} \frac{1}{\left(1+\frac{v^{2}}{u^{2}}\right)^{2}}\right) .
$$

The expression inside curved parenthesis in last display, is a Riemann sum for the integral

$$
\frac{1}{2} \int\left(1+x^{2}\right)^{-2} d x=\frac{\pi}{4} .
$$

The error can be estimated with the trapezoid rule:

$$
\left|\frac{1}{b-a} \int_{a}^{b} f(x) d x-\frac{f(a)+f(b)}{2}\right| \leq \frac{(b-a)^{2}}{6} \sup _{a \leq x \leq b}\left|f^{\prime \prime}(x)\right|,
$$


which shows that we can write

$$
\left\|\nabla \psi_{f}\right\|^{2}=\sum_{k \in \mathbb{Z}}\left(\pi|k|+\frac{a(k)}{|k|}\right)|\widehat{f}(k)|^{2}
$$

for some bounded function $a: \mathbb{Z} \rightarrow \mathbb{R}$.

Given this lemma, in order to prove Theorem 2.8 we just need to identify the expression $\pi|k|+\frac{a(k)}{|k|}$ as the symbol in Fourier space of an operator of the form $-\frac{1}{2}(-\Delta)^{1 / 2}+K$, with $K$ an integral operator. The symbol of $-\frac{1}{2}(-\Delta)^{1 / 2}$ is exactly equal to $\pi|k|$, so the symbol of $K$ is equal to $\frac{a(k)}{|k|}$. Since $\frac{a(k)}{|k|} \in \ell^{2}$, there exists a function $K(x) \in L^{2}(\mathbb{T})$ such that

$$
K f(x)=\int K(y-x) f(y) d y
$$

and $K$ is a convolution operator.

\section{Acknowledgements}

PG thanks FCT/Portugal for support through the project UID/MAT/04459/ 2013. This project has received funding from the European Research Council (ERC) under the European Union's Horizon 2020 research and innovative programme (grant agreement No 715734).

\section{References}

S. Assing. A limit theorem for quadratic fluctuations in symmetric simple exclusion. Stochastic Process. Appl. 117 (6), 766-790 (2007). MR2327838.

O. Blondel, P. Gonçalves and M. Simon. Convergence to the stochastic Burgers equation from a degenerate microscopic dynamics. Electron. J. Probab. 21, Paper No. 69, 25 (2016). MR3580035.

I. Corwin. The Kardar-Parisi-Zhang equation and universality class. Random Matrices Theory Appl. 1 (1), 1130001, 76 (2012). MR2930377.

A. De Masi, N. Ianiro, A. Pellegrinotti and E. Presutti. A survey of the hydrodynamical behavior of many-particle systems. In Nonequilibrium phenomena, II, Stud. Statist. Mech., XI, pages 123-294. North-Holland, Amsterdam (1984). MR757003.

J. Diehl, M. Gubinelli and N. Perkowski. The Kardar-Parisi-Zhang equation as scaling limit of weakly asymmetric interacting Brownian motions. Comm. Math. Phys. 354 (2), 549-589 (2017). MR3663617.

P. A. Ferrari, E. Presutti and M. E. Vares. Nonequilibrium fluctuations for a zero range process. Ann. Inst. H. Poincaré Probab. Statist. 24 (2), 237-268 (1988). MR953119.

T. Franco, P. Gonçalves and M. Simon. Crossover to the stochastic Burgers equation for the WASEP with a slow bond. Comm. Math. Phys. 346 (3), 801-838 (2016). MR3537337.

P. Gonçalves and M. Jara. Scaling limits of additive functionals of interacting particle systems. Comm. Pure Appl. Math. 66 (5), 649-677 (2013). MR3028483. 
P. Gonçalves and M. Jara. Nonlinear fluctuations of weakly asymmetric interacting particle systems. Arch. Ration. Mech. Anal. 212 (2), 597-644 (2014). MR3176353.

P. Gonçalves, M. Jara and S. Sethuraman. A stochastic Burgers equation from a class of microscopic interactions. Ann. Probab. 43 (1), 286-338 (2015). MR3298474.

P. Gonçalves, M. Jara and M. Simon. Second order Boltzmann-Gibbs principle for polynomial functions and applications. J. Stat. Phys. 166 (1), 90-113 (2017). MR3592852.

P. Gonçalves, N. Perkowski and M. Simon. Derivation of the stochastic burgers equation with Dirichlet boundary conditions from the wasep $(2019+)$. To appear in Ann. Henri Lesbesgue.

M. Hairer. Solving the KPZ equation. Ann. of Math. (2) 178 (2), 559-664 (2013). MR3071506.

M. Hairer. A theory of regularity structures. Invent. Math. 198 (2), 269-504 (2014). MR3274562.

I. Mitoma. Tightness of probabilities on $C\left([0,1] ; \mathcal{S}^{\prime}\right)$ and $D\left([0,1] ; \mathcal{S}^{\prime}\right)$. Ann. Probab. 11 (4), 989-999 (1983). MR714961.

R. Rhodes and V. Vargas. Gaussian multiplicative chaos and applications: a review. Probab. Surv. 11, 315-392 (2014). MR3274356.

H. Spohn. Stochastic integrability and the KPZ equation. IAMP Bulletin pages 5-9 (2012). 